\title{
PHYTOCLIMATIC SPECTRUM OF WEEDS FLORA OF TOORMANG VALLEY, DIR LOWER,KOH-E-HINDUKUSH RANGE, PAKISTAN
}

\author{
Muhammad Idrees ${ }^{1}$, Wisal Muhammad Khan ${ }^{1 *}$, Haroon Khan ${ }^{2}$, Arshad Iqbal ${ }^{1}$, Nosheen \\ Umar $^{3}$, Shah Khalid ${ }^{1}$ and Nisar Ahmad ${ }^{4}$
}

DOI: https://doi.org/10.28941/pjwsr.v27i4.957

\begin{abstract}
Four season's data of floristic structure and biological spectrum of Toormang Valley, Dir lower was explored during 2018-2019. The flora comprised 238 species, 164 genera associated with 60 families. The most prevailing family was Asteraceae with 42 species (17.64\%), trailed by Rosaceae $16(6.72 \%)$, Brassicaceae 13(5.46\%), Solanaceae $11(4.62 \%)$, Papilionaceae 10 (4.20\%), Apiaceae, and Poaceae each with 9 $(3.78 \%)$, Lamiaceae 8 (3.36\%), Boraginaceae, Euphorbiaceae and Moraceae each contributed by 7 species (2.94\%), Amaranthaceae and Cucurbitaceae each consisted of 6 species $(2.52 \%)$, Caryophyllaceae and Chenopodiaceae each with $5(2.10 \%)$ while rest of 23 families contributed by 1 species each (0.42\%). The largest genera were Euphorbia ( 6 species), followed by Sonchus and Medicago (4 species) each. Therophytes were the dominant with 102 species $(42.85 \%)$, followed by nanophanerophytes with 27 $(11.34 \%)$, hemicryptophytes with $25(10.50 \%)$, chamaephytes with $20(8.40 \%)$, microphanerophytes with $18(7.56 \%)$, megaphanerophytes with $16(6.72 \%)$, geophytes with $15(6.30 \%)$, mesophanerophytes with $14(5.88 \%)$ and parasite with 1 species $(0.42 \%)$. The observations on leaf size revealed that microphyll was the prevailing class with 73 species $(30.67 \%)$, followed by mesophyll $69(28.99 \%)$, nanophyll $60(25.21 \%)$, leptophyll $23(9.66 \%)$, macrophyll $8(3.36 \%)$, megaphylly $4(1.1 .68 \%)$ and aphyllous with 1 species $(0.42 \%)$. Simple lamina species 154 while 5 species had spiny lamina shape.
\end{abstract}

Keywords: Weeds flora, phytoclimatic spectrum, Toormang valley

Citation: Idrees, M.; W.M. Khan; H. Khan; A. Iqbal; N. Umar; S. Khalid and N. Ahmad. 2021. Phytoclimatic Spectrum of Weeds Flora of Toormang Valley, Dir Lower,Koh-eHindukush Range, Pakistan. Pak. J. Weed Sci. Res., 27(4): 451-473.

\footnotetext{
${ }^{1}$ Department of Botany, Islamia College Peshawar, Pakistan

${ }^{2}$ Department of Weed Science \& Botany, The University of Agriculture,Peshawar, Pakistan ${ }^{3}$ Department of Botany, University of Peshawar, Pakistan

${ }^{4}$ Department of Biotechnology, University of Science and Technology Bannu.

*Corresponding author's email: wisalbot@icp.edu.pk
} 


\section{INTRODUCTION}

The Valley is situated in Lower Dir, in the Northwest of Khyber Pakhtunkhwa, Pakistan, and is restricted by a vast series of Koh-e-Hindukush Range. It is situated between 34-37 to 35-7 North latitude and 71-31 to 72-14 East longitudes. The Valley is bounded by Wari in the North, Khall, and Rabat in the West, Laram hills in the South, and Swat in the East. The climate of the Valley is influenced by different ecological and topographic features. The high peaks of the mountains receive snowfall during December, January, and February. The summer is pleasant and short while winter is the harsh season of the year. Agriculture and forests are the main sources of the economy of the indigenous peoples. Flora is the plant species of a given geographical area, whereas vegetation is an ecological term anddetermines the plant population, size, distribution, andrelative importance (RI). (Ali, 2008). Changing environmental situations such as acid rain, soil erosion are alarming threats to flora (Hussain, 2003) while, on the other hand, the floristic configuration is a consideration of phytodiversity, environmental and biotic effects. So, studies on the local flora constantly provide accurate information. Leaf size and life form spectrum are key physiognomic characteristics commonly used in the analysis of vegetation. The biological spectrumdetermines the micro and macroclimatic conditions of a geographical area (Shimwell, 1971). Many studies have been described therophytes as indicator species of a particular desert-type climate (Samreen et al., 2016). Shah et al. (2013) reported the floristic characteristics of each species from a humid forest situated in an inaccessible area of Pakistan. Hussain et al. (2015) conducted research work on Phytodiversity and ecological physiognomies of the flora of Mastuj, Chitral, and reported the highest percentage of therophytes followed by hemicryptophytes and geophytes. Seraj et al. (2014) determined the Raunkiaerian life form at Asir Mountain of Saudi Arabia and described that therophytes were the prevailing class followed by chaemophytes. Alsherif et al. (2013) prepared the Raunkierian life form of the Khuliais area, Saudi Arabia, and found that therophytes were the dominant class with the highest percentage of species followed by chaemophytes and hemicryptophytes. Khan et al. (2012) reported that leaf spectra of plants study show the dominance of microphyll followed by nanophyll and leptophyll from Tehsil Takht-e-Nasrati, Karak, Pakistan. The highest percentage of microphyll followed by leptophyll, nanophyll \& megaphyll shows that the studied area is under severe biotic stress due to overgrazing and deforestation (Sher and Khan, 2007). The literature study revealed that the research area is virgin regarding its floristic and ecological characteristics.

\section{MATERIALS AND METHODS}

Four season's floristic data of the valley was collected during the year 2018-2019 by walking method (Nazar et al., 2008). Plant specimens were collected, pressed, dried, and mounted on standard herbarium sheets. Plants were identified with the help of Flora of Pakistan (Nasir and Ali, 1970-1989; Ali and Nasir, 1989-1992; Ali and Qaiser, 1995-2015) and verifiedfrom the Herbarium, University of Malakand. An alphabetical family-wise list of plants was prepared. The voucher specimens were submitted to the herbarium, Department of Botany, UOM. The tools used during research work were a knife, polythene bags, old newspapers, plant presser, map of the area, notebook, and pencil.

\section{RESULTS AND DISCUSSION Floristic and ecological attributes}

The study of the floristic composition of vegetation is crucial for conservation management by providing habitats for wildlife and contributing to the ecologically sustainable management of natural resources (Ahmad and Ehsan, 2012). The flora of the Valley consisted of 238 species, 164 genera from sixty (60) families. It comprisedof 53 dicots, 4 monocots, 01 gymnosperm, and 2 pteridophyte families. Pteridophyte had 3 genera $(1.82 \%)$ while, gymnosperms has 1 genus $(0.60 \%)$. There were 11 
genera of monocots (6.70\%) and 149 genera of dicots (90.85\%). Leading families were Asteraceae 42 species $(17.64 \%)$ followed by Rosaceae 16 species $(6.72 \%)$, Brassicaceae 13 species $(5.46 \%)$, Solanaceae 11 species $(4.62 \%)$, Papilionaceae 10 species $(4.20 \%)$, Apiaceae, and Poaceae each with 9 species $(3.78 \%)$, followed by Lamiaceae contributed by 8 species $(3.36 \%)$. Boraginaceae, Euphorbiaceae, and Moraceae each contributed by 7 species (2.94\%) while, Amaranthaceae and Cucurbitaceae each consisted of 6 species $(2.52 \%)$. Caryophyllaceae and Chenopodiaceae had 5 species $(2.10 \%)$ each, Aspleniaceae and Salicaceae each consists of 4 species $(1.68 \%)$ while Fagaceae, Oleaceae, Ranunculaceae, Rutaceae, and Urticaceae each contributed by 3 species (1.26\%), which is followed by Alliaceae, Asclepiadaceae, Cannabaceae, Ebenaceae, Fumariaceae, Malvaceae, Mimosaceae, Pinaceae, Polygonaceae, and Zygophyllaceae contributed 2 species each $(0.84 \%)$. The rest of the 23 families contributed by 1 species each $(0.42 \%)$. (Table 3, Fig. 2) The current reportsmatch with Haq et al. (2010) and Ravanbakhsh et al. (2014) who describedthat the Asteraceae and Rosaceae are theleading families in their research geographical zone. Similarly, many other authors also documented that Asteraceae was the leading family in their research zones (Ali et al., 2016; Badshah et al., 2016; Inayat et al., 2014; Khan et al., 2011, 2013, 2014; Ganji, 2016; Hussain et al., 2015; Shah et al., 2013; Sher et al., 2014; Ullah and Ullah, 2016). The flora of the research area included wild $196(82.35 \%)$ and cultivated plant species 42 (17.64\%). The largest genera were Euphorbia (6 species) followed by Sonchus, Chenopodium, and Pyrus (4 species each). There were 5 thorny species $(2.10 \%)$ and $233(97.89 \%)$ non-thorny species. Based on habitat 115 species $(48.31 \%)$ were found in dry conditions, 35 species $(17.64 \%)$ in wet, 42 species $(17.64 \%)$ in cultivated, and 46 species $(19.32 \%)$ in both dry and wet conditions. The observations on leaf size revealed that the most prevailing class was microphyll with 73 species $(30.67 \%)$ followed by mesophyll 69 species (28.99\%), nanophyll 60 species $(25.21 \%)$, leptophyll 23 species $(9.66 \%)$, macrophyll 8 species $(3.36 \%)$, megaphylly 4 species $(1.1 .68 \%)$ and aphyllous contributed by 1 species $(0.42 \%)$. The majority of the species $154(64.70 \%)$ had simple lamina while 5 species have spiny lamina shape. The study area has four distinct seasons with mild summer and harsh winter. The seasonal distinction in flora was verified, with the highest species richness in the summer and spring seasons. Summer flora had 135 species (30.57\%), spring with 182 species (30.08\%), and autumn had 130 species $(21.48 \%)$. The lowest species richness 108 (17.35\%) were recorded in winter. Our results are strongly correlated with the findings of other researchers such as; Ullah and Badshah (2017) reported the highest number of species in the summer season from Jelar Valley Dir Upper. Similarly, the highest percentage of species were recorded in the spring and summer seasons from Chail Valley Swat and Darazinda D.I. Khan (Ali et al., 2016; Samreen et al., 2016).

\section{Biological spectrums}

The phytoclimate determines the biological spectrum of the vegetation of a geographical zone (Khan et al., 2013). The life form of plant species of an area reflectstolerance towards the climatic variations and their genetic makeup (Hussain et al., 2015). The characteristics of the flora and vegetation are reflected by the biological spectra and environmental gradients. Raunkiaer (1934) suggestedclassification of the life form based onthe position of perennating buds during unfavorable environmental conditions. Using this criterion, it was observed that therophytes werethe most abundant life with 102 species (42.85\%) followed by nanophanerophytes represented by 27 species $(11.34 \%)$, hemicryptophyte with 25 species $(10.50 \%)$, and Chamaephytes having 20 species $(8.40 \%)$. Microphanerophytes were represented by 18 species $(7.56 \%)$, megaphanerophytes with 16 species $(6.72 \%)$, geophytes with 15 species (6.30), mesophanerophytes with 14 species $(5.88 \%)$, and parasite 1 species $(0.42)$ stayed the next prevailing life 
forms. (Table 5, Fig. 3). Badshah et al. (2016) and Naveed et al. (2012) reported that therophytes and nanophanerophytes were the dominant life form classes in their study areas. The dominance of therophytes in this area is due to extreme climatic conditions, overgrazing, and human interference.(Rafay et al., 2013).

\section{Seasonal variation}

The current observations show that the highest number of plants species (185) were found in the summer season with therophytes, 64 species $(34.59 \%)$, the most prevailing life form, followed by nanophanerophytes with 24 $(12.97 \%)$, hemicryptophytes with 21 species (11.35\%), Chamaephytes and microphanerophytes each with 18 species $(9.72 \%)$, megaphanerophytes with 16 species each 8.64\%), mesophanerophytes with 14 species $(7.56 \%)$, geophytes with 14 species $(7,56 \%)$, and parasite were represented by 1 species $(0.54 \%)$. Therophytes were also dominant in the spring season represented by 58 species (32.95\%), followed by nanophanerophytes 25 species $(14.20 \%)$, microphanerophytes 18 species (10.22\%), hemicrophanerophytes 17 species (9.65\%), megaphanerophytes 16 species $(9.90 \%)$, Chamaephytes 15 species $(8.52 \%)$, mesophanerophytes 14 species $(7.95 \%)$, geophytes 12 species $(6.81 \%)$, and parasite represented by 1 species $(0.56 \%)$. During Autumn therophytes consist of 31 species (24.03\%), nanophanerophytes 23 species (17.82\%), microphanerophytes 18 species (13.95\%), megaphanerophytes 16 species $(12.40 \%)$, mesophanerophytes 14 species $(11.11 \%)$, hemicryptophytes 13 species $(10.07 \%)$, Chamaephytes 11 species $(8.52 \%)$, geophytes 2 species $(1.55 \%)$, and parasite include 1 species $(0.77 \%)$, while, the lowest numbers of species were noted in winter. (Table 1, Fig. 4). Our currentinvestigations are supported by Badshah et al. (2016) who described that therophytes werethe highest in number during spring and summer seasons from Parachinar, Kurram Agency, Pakistan.

\section{Leaf size spectrum}

The observation of the leaf size explains the plant's biological processes and their communities. Leaf size and biological spectra are used in theclassification and association of communities (Ali et al., 2016). It is useful for the understanding of physiological processes of plant species and their communities (Oosting, 1956) as well as the leaf-sized classes are important for studying the associations. Our findings on leaf size exhibited that macrophyll was the most leading class with 73 species $(30.68 \%)$, followed by mesophyll 69 species (28.99\%), nanophyll 60 species $(25.22 \%)$, leptophyll 23 species $(9.66 \%)$, macrophyll 8 species $(1.26 \%)$, megaphyll 4 species $(1.68 \%)$, and aphyllous comprised by 1 species of Cuscuta reflexa Roxb, $(0.42 \%)$. (Table 5, Fig.7). Our results are in line with the findings of Khan et al. (2013), Shah et al. (2013), Khan et al. (2011), Amjad. (2012) and Khan et al. (2014).

\section{Seasonal variation}

In summer, mesophyll was the prevailing leaf size class contributed by 58 species $(31.35 \%)$, followed by microphyll 55 species $(29.72 \%)$, nanophyll 48 species $(25.94 \%)$, leptophyll 18 species $(9.72 \%)$, macrophyll 4 species $(2.16 \%)$, megaphyll and aphyllous by 1 species each $(0.54 \%)$. In spring microphyll were dominant form consisted of 53 species $(30.81 \%)$, followed by mesophyll 49 species $(28.48 \%)$, nanophyll 44 species (25.58\%), leptophyll 13 species (7.55\%), macrophyll 8 species $(4.65 \%)$, megaphyll 4 species $(2.32 \%)$, and aphyllous contributed by 1 species $(0.58 \%)$. In autumn mesophyll was the dominant form consisted of 45 species $(34.88 \%)$, followed by nanophyll 33 species $(25.58 \%)$, microphyll 31 species $(24.03 \%)$, leptophyll 14 species $(10.85 \%)$, macrophyll 3 species $(2.32 \%)$, megaphyll 2 species $(1.55 \%)$, and aphyllous contributed by 1 species $(0.77 \%)$. While mesophyll was also dominant in winter contributed by 37 species (33.94\%), followed by microphyll with 32 species (29.35\%), nanophyll with 24 species $(22.01 \%)$, leptophyll with 8 species $(7.33 \%)$, macrophyll with 6 species $(5.50 \%)$, 
megaphyll and aphyllous contributed by 1 species each $(0.91 \%)$. (Table 2 , Fig. 5).

\section{Lamina shape}

The shape of leaf lamina of different plant species revealed that 154 species $(64.70 \%)$ have simple lamina shape, followed by compound and dissected lamina each have 37 species $(15.54 \%)$, spiny 5 species $(2.10 \%)$, needles lamina shape consist 2 species $(0.84 \%)$, while in 3 species lamina shape is absent. (Table 5, Fig. 6) The lamina shapes of the plant species were also reported from Jelar Valley, Upper Dir(Ullah and Badshah., 2017), from Darazinda DI. Khan (Samreen et al., 2016) and from Chail Valley, Swat. (Ali et al., 2016).

\section{Habit and Habitat}

Among the flora, herbs were dominant comprises 167 species $(70.16 \%)$, followed by shrub 48 species $(18.06 \%)$ and trees were contributed by 28 species $(11.76 \%$ ). (Table 5, Fig. 9) The highest numbers of 115 species $(48.31 \%)$ were found growing in dry conditions, followed by 46 species $(19.32 \%)$ growing in both dry and wet conditions, 42 species were found growing on agricultural land, while 35 species were found growing on the wetland. (Table 5, Fig. 8). The research area is under high anthropogenic pressure as indigenous peoples rely on fuelwood and forest timber. Ultimately, this rapid deforestation resulting indisturbing themico and micro-habitats (Ali et al., 2016).

\section{Conclusion}

The present results revealed that the flora of the valley consisted of 238 species, 164 genera from 60 families. It included 53 dicots, 04 monocot families, 01 family of Gymnosperms, and 2 Pteridophyte families. Pteridophyte had 3 genera (1.82\%) while; Gymnosperms had 01 genus $(0.60 \%)$. There were 11 genera of monocots (6.70\%) and 149 genera of dicots (90.85\%). Leading families were Asteraceae 42 species $(17.64 \%)$, Rosaceae 16 species $(6.72 \%)$, Brassicaceae 13 species $(5.46 \%)$, Solanaceae 11 species $(4.62 \%)$, and Papilionaceae 10 species $(4.20 \%)$. The largest genera regarding the number were Euphorbia (6 species), Sonchus, Chenopodium, Medicago, and Pyrus (4 species each). The dominant life form based on species richness was therophytes with 102 species (42.85\%), followed by nanophanerophytes with 27 species (11.34\%). Leaf size spectra showed that the macrophyll was the most dominant leaf size classwith 73 species $(30.67 \%)$, followed by mesophyll 69 species (28.99\%). The current study provides baseline observations and information of Toormang flora and further study is recommended for the exploration of quantitative analysis of the vegetation. 
456 Muhammad Idrees, Wisal Muhammad Khan et al. Phytoclimatic Spectrum ....

Table 1: Seasonal variation in the life form

\begin{tabular}{|c|c|c|c|c|c|c|c|c|c|}
\hline $\begin{array}{l}\text { S.N } \\
\text { O. }\end{array}$ & Life form & $\begin{array}{c}\text { Sprin } \\
\mathbf{g}\end{array}$ & \%age & $\begin{array}{c}\text { Summ } \\
\text { er }\end{array}$ & \%age & \begin{tabular}{|c|} 
Autum \\
$\mathrm{n}$
\end{tabular} & \%age & $\begin{array}{c}\text { Winte } \\
r\end{array}$ & \%age \\
\hline 1. & Therophytes & 58 & $\begin{array}{c}32.95 \\
\%\end{array}$ & 64 & $\begin{array}{c}34.59 \\
\%\end{array}$ & 31 & $\begin{array}{c}24.03 \\
\%\end{array}$ & 22 & $\begin{array}{c}20.72 \\
\%\end{array}$ \\
\hline 2. & $\begin{array}{l}\text { Hemicryptophyte } \\
\mathrm{s}\end{array}$ & 17 & $9.65 \%$ & 21 & $\begin{array}{c}11.35 \\
\%\end{array}$ & 13 & $\begin{array}{c}10.07 \\
\%\end{array}$ & 4 & $3.77 \%$ \\
\hline 3. & $\begin{array}{l}\text { Nanophanerophy } \\
\text { tes }\end{array}$ & 25 & $\begin{array}{c}14.20 \\
\%\end{array}$ & 24 & $\begin{array}{c}12.97 \\
\%\end{array}$ & 23 & $\begin{array}{c}17.82 \\
\%\end{array}$ & 18 & $\begin{array}{c}16.98 \\
\%\end{array}$ \\
\hline 4. & Chamaephytes & 15 & $8.52 \%$ & 18 & $9.72 \%$ & 11 & $8.52 \%$ & 9 & $8.49 \%$ \\
\hline 5. & $\begin{array}{l}\text { Microphanerophy } \\
\text { tes }\end{array}$ & 18 & $\begin{array}{c}10.22 \\
\%\end{array}$ & 18 & $9.72 \%$ & 18 & $\begin{array}{c}13.95 \\
\%\end{array}$ & 17 & $\begin{array}{c}16.03 \\
\%\end{array}$ \\
\hline 6. & $\begin{array}{l}\text { Megaphanerophy } \\
\text { tes }\end{array}$ & 16 & $9.09 \%$ & 16 & $8.64 \%$ & 16 & $\begin{array}{c}12.40 \\
\%\end{array}$ & 16 & $\begin{array}{c}15.09 \\
\%\end{array}$ \\
\hline 7. & Geophytes & 12 & $6.81 \%$ & 9 & $4.86 \%$ & 2 & $1.55 \%$ & 5 & $4.71 \%$ \\
\hline 8. & $\begin{array}{l}\text { Mesophanerophy } \\
\text { tes }\end{array}$ & 14 & $7.95 \%$ & 14 & $7.56 \%$ & 14 & $\begin{array}{c}11.11 \\
\%\end{array}$ & 14 & $\begin{array}{c}13.20 \\
\%\end{array}$ \\
\hline 9. & Parasite & 1 & $0.56 \%$ & 1 & $0.54 \%$ & 1 & $0.77 \%$ & 1 & $0.94 \%$ \\
\hline & & 176 & & 185 & & 129 & & 106 & $\begin{array}{c}99.93 \\
\%\end{array}$ \\
\hline
\end{tabular}

Table 2: Seasonal variation of Leaf size spectra

\begin{tabular}{|l|c|c|c|c|c|c|c|c|}
\hline $\begin{array}{l}\text { Leaf size } \\
\text { spectra }\end{array}$ & Spring & $\mathbf{\%}$ & Summer & $\mathbf{\%}$ & Autumn & $\mathbf{\%}$ & Winter & $\%$ \\
\hline Microphyll & 53 & $30.81 \%$ & 55 & $29.72 \%$ & 31 & $24.03 \%$ & 32 & $29.35 \%$ \\
\hline Mesophyll & 49 & $28.48 \%$ & 58 & $31.35 \%$ & 45 & $34.88 \%$ & 37 & $33.94 \%$ \\
\hline Nanophyll & 44 & $25.58 \%$ & 48 & $25.94 \%$ & 33 & $25.58 \%$ & 24 & $22.01 \%$ \\
\hline Leptophyll & 13 & $7.55 \%$ & 18 & $9.72 \%$ & 14 & $10.85 \%$ & 8 & $7.33 \%$ \\
\hline Macrophyll & 8 & $4.65 \%$ & 4 & $2.16 \%$ & 3 & $2.32 \%$ & 6 & $5.50 \%$ \\
\hline Megaphyll & 4 & $2.32 \%$ & 1 & $0.54 \%$ & 2 & $1.55 \%$ & 1 & $0.91 \%$ \\
\hline Aphyllous & 1 & $0.58 \%$ & 1 & $0.54 \%$ & 1 & $0.77 \%$ & 1 & $0.91 \%$ \\
\hline & 172 & $99.97 \%$ & 185 & & 129 & $99.98 \%$ & 109 & $99.95 \%$ \\
\hline
\end{tabular}

Table 3: Floristic list and ecological characteristic of flora of the valley

\begin{tabular}{|c|c|c|c|c|c|c|c|c|c|c|c|}
\hline \multirow{2}{*}{$\begin{array}{l}\text { S. } \\
\text { No } \\
\text {. }\end{array}$} & \multirow{2}{*}{ Species } & \multirow{2}{*}{ Family } & \multirow{2}{*}{$\begin{array}{l}\text { Ha } \\
\text { bit }\end{array}$} & \multirow{2}{*}{$\begin{array}{l}\text { Habit } \\
\text { at }\end{array}$} & \multirow{2}{*}{$\begin{array}{c}\text { Life } \\
\text { For } \\
\text { m }\end{array}$} & \multirow{2}{*}{\begin{tabular}{|c|} 
Leaf \\
Size \\
Spectru \\
m
\end{tabular}} & \multirow{2}{*}{$\begin{array}{c}\text { Lamin } \\
\text { a } \\
\text { Shape }\end{array}$} & \multicolumn{4}{|c|}{$\begin{array}{c}\text { Seasonalit } \\
y\end{array}$} \\
\hline & & & & & & & & A & $\mathbf{w}$ & $\begin{array}{l}\mathbf{S} \\
\mathbf{P}\end{array}$ & SM \\
\hline 1. & $\begin{array}{l}\text { Alisma plantago- } \\
\text { aquatica L. }\end{array}$ & Alismataceae & $\mathrm{H}$ & W & Hem & $\mathrm{N}$ & $\mathrm{S}$ & - & - & - & + \\
\hline 2. & $\begin{array}{l}\text { Sagittaria } \\
\text { trifolia. L. }\end{array}$ & Alismataceae & $\mathrm{H}$ & W & G & Mes & Dis & - & - & + & + \\
\hline 3. & $\begin{array}{l}\text { Allium sativum } \\
\text { L. }\end{array}$ & Alliaceae & $\mathrm{H}$ & $\mathrm{Cu}$ & G & Mes & $\mathrm{S}$ & - & - & + & - \\
\hline 4. & Allium cepa L. & Alliaceae & $\mathrm{H}$ & $\mathrm{Cu}$ & G & Mes & $\mathrm{S}$ & - & - & + & - \\
\hline 5. & $\begin{array}{l}\text { Narcissus tazetta } \\
\text { L. }\end{array}$ & $\begin{array}{l}\text { Amaryllidacea } \\
\text { e }\end{array}$ & $\mathrm{H}$ & D & G & Mes & S & - & + & - & - \\
\hline 6. & $\begin{array}{l}\text { Colocasia } \\
\text { esculenta (L.) } \\
\text { Schott. }\end{array}$ & Araceae & $\mathrm{H}$ & $\mathrm{Cu}$ & G & Meg & $\mathrm{S}$ & - & + & + & - \\
\hline 7. & Avena sativa $\mathrm{L}$. & Poacaea & $\mathrm{H}$ & $\mathrm{D}$ & Th & Mic & $\mathrm{S}$ & - & - & + & - \\
\hline 8. & $\begin{array}{l}\text { Cymbopogon } \\
\text { commutatus } \\
\text { (Steud.) Stapf. }\end{array}$ & Poaceae & $\mathrm{H}$ & WD & Ch & Mic & $\mathrm{S}$ & + & + & + & + \\
\hline 9. & $\begin{array}{l}\text { Cynodon } \\
\text { dactylon (L.) } \\
\text { Pers. }\end{array}$ & Poaceae & $\mathrm{H}$ & WD & Hem & Mic & $S$ & + & + & + & + \\
\hline
\end{tabular}




\begin{tabular}{|c|c|c|c|c|c|c|c|c|c|c|c|}
\hline \multirow{2}{*}{$\begin{array}{l}\text { S. } \\
\text { No }\end{array}$} & \multirow{2}{*}{ Species } & \multirow{2}{*}{ Family } & \multirow{2}{*}{$\begin{array}{l}\text { Ha } \\
\text { bit }\end{array}$} & \multirow{2}{*}{$\begin{array}{c}\text { Habit } \\
\text { at }\end{array}$} & \multirow{2}{*}{$\begin{array}{l}\text { Life } \\
\text { For } \\
\text { m }\end{array}$} & \multirow{2}{*}{$\begin{array}{c}\text { Leaf } \\
\text { Size } \\
\text { Spectru } \\
\text { m }\end{array}$} & \multirow{2}{*}{$\begin{array}{c}\text { Lamin } \\
\text { a } \\
\text { Shape }\end{array}$} & \multicolumn{4}{|c|}{$\begin{array}{c}\text { Seasonalit } \\
y \\
\end{array}$} \\
\hline & & & & & & & & A & $\mathbf{W}$ & $\begin{array}{l}\mathbf{S} \\
\mathbf{P}\end{array}$ & SM \\
\hline 10. & $\begin{array}{l}\text { Hordeum } \\
\text { murinum L. }\end{array}$ & Poaceae & $\mathrm{H}$ & $\mathrm{Cu}$ & $\mathrm{Ch}$ & $\mathrm{Np}$ & S & - & + & - & + \\
\hline 11. & $\begin{array}{l}\text { Hordeum } \\
\text { vulgare L. }\end{array}$ & Poaceae & $\mathrm{H}$ & $\mathrm{Cu}$ & Hem & Mic & S & - & - & + & + \\
\hline 12. & Oryza sativa L. & Poaceae & $\mathrm{H}$ & $\mathrm{Cu}$ & G & Mic & $\mathrm{S}$ & - & - & + & + \\
\hline 13. & $\begin{array}{l}\text { Sorghum } \\
\text { halepense (L.) } \\
\text { Pers }\end{array}$ & Poaceae & $\mathrm{H}$ & $\mathrm{D}$ & Hem & Mic & S & - & - & + & + \\
\hline 14. & $\begin{array}{l}\text { Triticum } \\
\text { aestivum L. }\end{array}$ & Poaceae & $\mathrm{H}$ & $\mathrm{Cu}$ & Th & Mic & S & - & + & - & + \\
\hline 15. & Zea mwys L. & Poaceae & $\mathrm{S}$ & $\mathrm{Cu}$ & Th & Mes & $\mathrm{S}$ & + & - & - & + \\
\hline 16. & $\begin{array}{l}\text { Justicia } \\
\text { adhatoda (L.) } \\
\text { Huth. }\end{array}$ & Acanthaceae & S & W & $\mathrm{Np}$ & Mes & $S$ & + & + & + & + \\
\hline 17. & $\begin{array}{l}\text { Strobilanthes } \\
\text { urticifolia Wall. } \\
\text { ex kuntze. }\end{array}$ & Acanthaceae & $\mathrm{H}$ & W & $\mathrm{Np}$ & $\mathrm{N}$ & $S$ & + & - & - & + \\
\hline 18. & $\begin{array}{l}\text { Adiantum } \\
\text { capillus-veneris } \\
\text { L. }\end{array}$ & Adianthaceae & $\mathrm{H}$ & W & G & $\mathrm{N}$ & Dis & + & + & + & + \\
\hline 19. & $\begin{array}{l}\text { Achyranthes } \\
\text { aspera L. }\end{array}$ & $\begin{array}{l}\text { Amaranthacea } \\
\text { e }\end{array}$ & $\mathrm{H}$ & $\mathrm{D}$ & Th & $\mathrm{N}$ & S & + & - & - & - \\
\hline 20. & $\begin{array}{l}\text { Alternanthera } \\
\text { sessilis (L) R.Br. } \\
\text { ex DC. }\end{array}$ & $\begin{array}{l}\text { Amaranthacea } \\
\text { e }\end{array}$ & $\mathrm{H}$ & $\mathrm{D}$ & Th & Mic & $S$ & - & - & + & - \\
\hline 21. & $\begin{array}{l}\text { Amaranthus } \\
\text { caudatus L. }\end{array}$ & $\begin{array}{l}\text { Amaranthacea } \\
\text { e }\end{array}$ & $\mathrm{H}$ & W & Th & Mic & $\mathrm{S}$ & + & - & - & + \\
\hline 22. & $\begin{array}{l}\text { Amaranthus } \\
\text { spinosus L. }\end{array}$ & $\begin{array}{l}\text { Amaranthacea } \\
\text { e }\end{array}$ & $\mathrm{H}$ & WD & Th & Mic & S & - & - & - & + \\
\hline 23. & $\begin{array}{l}\text { Amaranthus } \\
\text { viridis L. }\end{array}$ & $\begin{array}{l}\text { Amaranthacea } \\
\text { e }\end{array}$ & $\mathrm{H}$ & $\mathrm{D}$ & Th & $\mathrm{N}$ & $S$ & + & - & - & - \\
\hline 24. & $\begin{array}{l}\text { Celosia argentea } \\
\text { L.var. cristata } \\
\text { (L.) Schinz. }\end{array}$ & $\begin{array}{l}\text { Amaranthacea } \\
\text { e }\end{array}$ & $\mathrm{H}$ & WD & Th & $\mathrm{N}$ & $S$ & - & - & - & + \\
\hline 25. & $\begin{array}{l}\text { Ammi visnaga } \\
\text { (L) Lam. }\end{array}$ & Apiaceae & $\mathrm{H}$ & $\mathrm{D}$ & $\mathrm{Ch}$ & Lp & Dis & - & + & + & + \\
\hline 26. & $\begin{array}{l}\text { Coriandrum } \\
\text { sativum L. }\end{array}$ & Apiaceae & $\mathrm{H}$ & $\mathrm{Cu}$ & Th & Lp & Dis & + & - & - & + \\
\hline 27. & Daucus carota L. & Apiaceae & $\mathrm{H}$ & $\mathrm{Cu}$ & G & Mes & Comp & - & + & + & - \\
\hline 28. & $\begin{array}{l}\text { Eryngium } \\
\text { caeruleum } \\
\text { M.Bieb. }\end{array}$ & Apiaceae & $\mathrm{H}$ & $\mathrm{D}$ & Hem & $\mathrm{N}$ & S & + & - & + & - \\
\hline 29. & $\begin{array}{l}\text { Eryngium } \\
\text { bourgatii Gouan. }\end{array}$ & Apiaceae & $\mathrm{H}$ & D & $\mathrm{Np}$ & Meg & S & + & - & + & - \\
\hline 30. & $\begin{array}{l}\text { Foeniculum } \\
\text { vulgare Mill. }\end{array}$ & Apiaceae & $\mathrm{H}$ & $\mathrm{D}$ & Th & $\mathrm{N}$ & Dis & + & - & - & + \\
\hline 31. & $\begin{array}{l}\text { Seseli libanotis } \\
\text { (L.) W.D.J.Koch. }\end{array}$ & Apiaceae & $\mathrm{H}$ & $D$ & Th & Lp & Comp & + & - & - & + \\
\hline 32. & $\begin{array}{l}\text { Scandix pecten- } \\
\text { veneris L. }\end{array}$ & Apiaceae & $\mathrm{H}$ & WD & Th & Mic & Comp & - & + & + & - \\
\hline 33. & $\begin{array}{l}\text { Trachyspermum } \\
\text { ammi (L.) }\end{array}$ & Apiaceae & $\mathrm{H}$ & $D$ & Th & Lp & Comp & - & - & + & + \\
\hline
\end{tabular}


458 Muhammad Idrees, Wisal Muhammad Khan et al. Phytoclimatic Spectrum ....

\begin{tabular}{|c|c|c|c|c|c|c|c|c|c|c|c|}
\hline \multirow{2}{*}{$\begin{array}{l}\text { S. } \\
\text { No } \\
\end{array}$} & \multirow{2}{*}{ Species } & \multirow{2}{*}{ Family } & \multirow{2}{*}{$\begin{array}{l}\text { Ha } \\
\text { bit }\end{array}$} & \multirow{2}{*}{$\begin{array}{c}\text { Habit } \\
\text { at }\end{array}$} & \multirow{2}{*}{$\begin{array}{c}\text { Life } \\
\text { For } \\
\text { m }\end{array}$} & \multirow{2}{*}{$\begin{array}{c}\text { Leaf } \\
\text { Size } \\
\text { Spectru } \\
\text { m }\end{array}$} & \multirow{2}{*}{$\begin{array}{c}\text { Lamin } \\
\text { a } \\
\text { Shape }\end{array}$} & \multicolumn{4}{|c|}{\begin{tabular}{|c|}
$\begin{array}{c}\text { Seasonalit } \\
y\end{array}$ \\
\end{tabular}} \\
\hline & & & & & & & & A & $\mathbf{w}$ & $\begin{array}{l}\mathbf{S} \\
\mathbf{P}\end{array}$ & SM \\
\hline & Sprague. & & & & & & & & & & \\
\hline 34. & $\begin{array}{l}\text { Nerium oleander } \\
\text { L. }\end{array}$ & Apocynaceae & S & $\mathrm{D}$ & $\mathrm{Np}$ & Mes & $S$ & + & + & + & + \\
\hline 35. & $\begin{array}{l}\text { Hedera } \\
\text { nepalensis K. } \\
\text { Koch. }\end{array}$ & Araliaceae & $\mathrm{H}$ & $D$ & $\mathrm{~Np}$ & Mes & $S$ & + & + & + & + \\
\hline 36. & $\begin{array}{l}\text { Phoenix } \\
\text { sylvestris (L.) } \\
\text { Roxb. }\end{array}$ & Aracaceae & $\mathrm{T}$ & WD & Mic & Mic & Comp & + & + & + & + \\
\hline 37. & $\begin{array}{l}\text { Asplenium } \\
\text { adiantum- } \\
\text { nigrum L. }\end{array}$ & Aspleniaceae & $\mathrm{H}$ & W & Hem & $\mathrm{N}$ & Comp & + & + & + & + \\
\hline 38. & $\begin{array}{l}\text { Asplenium } \\
\text { trichomanes L. }\end{array}$ & Aspleniaceae & $\mathrm{H}$ & W & Hem & Lp & Comp & + & + & + & + \\
\hline 39. & $\begin{array}{l}\text { Asplenium } \\
\text { septentrionale } \\
\text { (L.) Hoffm. }\end{array}$ & Aspleniaceae & $\mathrm{H}$ & W & Hem & Lp & Comp & + & - & + & + \\
\hline 40. & $\begin{array}{l}\text { Ceterach } \\
\text { dalhousiae } \\
\text { (Hook.) C. Chr. }\end{array}$ & Aspleniaceae & $\mathrm{H}$ & W & Hem & $\mathrm{N}$ & Comp & + & + & + & + \\
\hline 41. & $\begin{array}{l}\text { Calotropis } \\
\text { procera (Aiton.) } \\
\text { Dryand. }\end{array}$ & $\begin{array}{l}\text { Asclepiadacea } \\
\text { e }\end{array}$ & $S$ & $\mathrm{D}$ & $\mathrm{Np}$ & Mac & $S$ & + & + & + & + \\
\hline 42. & $\begin{array}{l}\text { Periploca aphylla } \\
\text { Decne. }\end{array}$ & $\begin{array}{l}\text { Asclepiadacea } \\
\text { e }\end{array}$ & $S$ & $\mathrm{D}$ & $\mathrm{Ch}$ & Ap & Abs & + & + & + & + \\
\hline 43. & $\begin{array}{l}\text { Achillea } \\
\text { millefolium L. }\end{array}$ & Asteraceae & $\mathrm{H}$ & $D$ & Hem & Lp & Dis & - & - & - & + \\
\hline 44. & $\begin{array}{l}\text { Anaphalis adnata } \\
\text { Wall.ex DC. }\end{array}$ & Asteraceae & $\mathrm{H}$ & WD & Th & Mes & $S$ & - & - & - & + \\
\hline 45. & $\begin{array}{l}\text { Artemisia } \\
\text { absinthium L. }\end{array}$ & Asteraceae & S & D & Th & $\mathrm{N}$ & Dis & + & - & - & + \\
\hline 46. & $\begin{array}{l}\text { Artemisia } \\
\text { scoparia Waldst. } \\
\text { \& Kitam. }\end{array}$ & Asteraceae & $\mathrm{H}$ & W & Th & $\mathrm{N}$ & Dis & + & - & - & + \\
\hline 47. & $\begin{array}{l}\text { Artemisia biennis } \\
\text { Willd. }\end{array}$ & Asteraceae & $\mathrm{H}$ & W & Hem & Mic & Dis & + & - & + & + \\
\hline 48. & $\begin{array}{l}\text { Calendula } \\
\text { arvensis M. Bieb. }\end{array}$ & Asteraceae & $\mathrm{H}$ & WD & Th & Mic & $S$ & - & - & + & - \\
\hline 49. & $\begin{array}{l}\text { Calendula } \\
\text { officinalis L. }\end{array}$ & Asteraceae & $\mathrm{H}$ & $\mathrm{D}$ & Th & Mes & S & + & + & + & + \\
\hline 50. & $\begin{array}{l}\text { Carduus } \\
\text { edelbergii } \\
\text { Rech.fil. } \\
\end{array}$ & Asteraceae & $\mathrm{H}$ & $\mathrm{D}$ & Th & Mac & $\mathrm{Sp}$ & - & + & + & - \\
\hline 51. & $\begin{array}{l}\text { Carthamus } \\
\text { oxyacantha } \\
\text { M.Bieb. }\end{array}$ & Asteraceae & $\mathrm{H}$ & $\mathrm{D}$ & Th & Mic & $S$ & - & - & + & - \\
\hline 52. & $\begin{array}{l}\text { Carthamus } \\
\text { tinctorius L. }\end{array}$ & Asteraceae & $\mathrm{H}$ & $\mathrm{D}$ & $\mathrm{Ch}$ & Mes & $S$ & - & - & + & - \\
\hline 53. & $\begin{array}{l}\text { Centaurea } \\
\text { calcitrapa L. }\end{array}$ & Asteraceae & $\mathrm{H}$ & D & Th & Mes & Dis & - & - & + & + \\
\hline 54. & $\begin{array}{l}\text { Chrysanthemum } \\
\text { cinerariaefolium }\end{array}$ & Asteraceae & $\mathrm{H}$ & WD & Th & Mic & S & - & - & + & - \\
\hline
\end{tabular}




\begin{tabular}{|c|c|c|c|c|c|c|c|c|c|c|c|}
\hline \multirow{2}{*}{$\begin{array}{l}\text { S. } \\
\text { No } \\
\text { - }\end{array}$} & \multirow{2}{*}{ Species } & \multirow{2}{*}{ Family } & \multirow{2}{*}{$\begin{array}{l}\text { Ha } \\
\text { bit }\end{array}$} & \multirow{2}{*}{$\begin{array}{c}\text { Habit } \\
\text { at }\end{array}$} & \multirow{2}{*}{$\begin{array}{l}\text { Life } \\
\text { For } \\
\text { m }\end{array}$} & \multirow{2}{*}{$\begin{array}{c}\text { Leaf } \\
\text { Size } \\
\text { Spectru } \\
\text { m }\end{array}$} & \multirow{2}{*}{$\begin{array}{l}\text { Lamin } \\
\text { a } \\
\text { Shape }\end{array}$} & \multicolumn{4}{|c|}{\begin{tabular}{|c|} 
Seasonalit \\
$y$
\end{tabular}} \\
\hline & & & & & & & & A & $\mathbf{w}$ & $\begin{array}{l}\mathbf{S} \\
\mathbf{P}\end{array}$ & SM \\
\hline & (Trevir.) Vis. & & & & & & & & & & \\
\hline 55. & $\begin{array}{l}\text { Cirsium falconeri } \\
\text { (Hook.f.) Petr. }\end{array}$ & Asteraceae & $\mathrm{H}$ & D & Hem & Mes & Sp & + & - & + & - \\
\hline 56. & $\begin{array}{l}\text { Conyza } \\
\text { bonariensis (L.) } \\
\text { Cronquist. }\end{array}$ & Asteraceae & $\mathrm{H}$ & W & Th & $\mathrm{N}$ & $S$ & + & - & - & + \\
\hline 57. & $\begin{array}{l}\text { Conyza } \\
\text { Canadensis (L.) } \\
\text { Cronquist. }\end{array}$ & Asteraceae & $\mathrm{H}$ & WD & Th & $\mathrm{N}$ & $S$ & - & - & - & + \\
\hline 58. & $\begin{array}{l}\text { Conyza } \\
\text { aegyptiaca (L.) } \\
\text { Dryand. ex } \\
\text { Aiton. }\end{array}$ & Asteraceae & $\mathrm{H}$ & $\mathrm{D}$ & $\mathrm{Ch}$ & Mes & S & + & - & - & + \\
\hline 59. & $\begin{array}{l}\text { Cosmos } \\
\text { bipinnatus Cav. }\end{array}$ & Asteraceae & $\mathrm{H}$ & D & Th & $\mathrm{N}$ & Comp & + & - & - & + \\
\hline 60. & $\begin{array}{l}\text { Erigeron } \\
\text { multicaulis Wall. } \\
\text { ex DC. }\end{array}$ & Asteraceae & $\mathrm{H}$ & $\mathrm{D}$ & Th & Mic & $S$ & - & - & - & + \\
\hline 61. & $\begin{array}{l}\text { Filago } \\
\text { hurdwarica } \\
\text { (Wall. ex DC.) } \\
\text { Wagenitz. }\end{array}$ & Asteraceae & $\mathrm{H}$ & $\mathrm{D}$ & Th & $\mathrm{N}$ & S & + & - & - & + \\
\hline 62. & $\begin{array}{l}\text { Galinsoga } \\
\text { parviflora Cav. }\end{array}$ & Asteraceae & $\mathrm{H}$ & $\mathrm{CU}$ & Th & Lp & $S$ & - & - & - & + \\
\hline 63. & $\begin{array}{l}\text { Gnaphalium } \\
\text { affine D. Don. }\end{array}$ & Asteraceae & $\mathrm{H}$ & $\mathrm{D}$ & Hem & Mes & $S$ & + & - & + & - \\
\hline 64. & $\begin{array}{l}\text { Helianthus } \\
\text { annuus L. }\end{array}$ & Asteraceae & $\mathrm{S}$ & $\mathrm{D}$ & Th & Mes & S & - & - & + & + \\
\hline 65. & $\begin{array}{l}\text { Lactuca serriola } \\
\text { L. }\end{array}$ & Asteraceae & $\mathrm{H}$ & WD & Th & Mes & Dis & - & - & + & + \\
\hline 66. & Lactuca sativa L. & Asteraceae & $\mathrm{H}$ & WD & Th & Mes & Dis & + & + & + & + \\
\hline 67. & $\begin{array}{l}\text { Launaea } \\
\text { nudicaulis (L.) } \\
\text { Hook.f. }\end{array}$ & Asteraceae & $\mathrm{H}$ & $\mathrm{D}$ & Hem & Mes & $S$ & - & - & - & + \\
\hline 68. & $\begin{array}{l}\text { Onopordum } \\
\text { acanthium L. }\end{array}$ & Asteraceae & $\mathrm{H}$ & $\mathrm{D}$ & Th & Mes & Dis & - & - & + & + \\
\hline 69. & $\begin{array}{l}\text { Parthenium } \\
\text { hysterophorus L }\end{array}$ & Asteraceae & $\mathrm{H}$ & WD & Mic & Mic & $S$ & + & - & + & + \\
\hline 70. & $\begin{array}{l}\text { Scorzonera } \\
\text { virgata DC. }\end{array}$ & Asteraceae & $\mathrm{H}$ & $\mathrm{D}$ & Th & Mes & $S$ & - & - & - & + \\
\hline 71. & $\begin{array}{l}\text { Senecio } \\
\text { chrysanthemoide } \\
\text { s DC. }\end{array}$ & Asteraceae & $\mathrm{H}$ & $\mathrm{D}$ & Th & Mes & $S$ & - & - & + & + \\
\hline 72. & $\begin{array}{l}\text { Serratula pallida } \\
\text { DC. }\end{array}$ & Asteraceae & $\mathrm{H}$ & WD & G & $\mathrm{N}$ & Dis & - & - & - & + \\
\hline 73. & $\begin{array}{l}\text { Sonchus } \\
\text { oleraceus (L.) L. }\end{array}$ & Asteraceae & $\mathrm{H}$ & WD & Th & Mic & Dis & - & - & - & + \\
\hline 74. & $\begin{array}{l}\text { Sonchus } \\
\text { auriculata L. }\end{array}$ & Asteraceae & $\mathrm{H}$ & $D$ & Th & Mic & Dis & - & - & - & + \\
\hline 75. & $\begin{array}{l}\text { Sonchus asper } \\
\text { (L.) Hill. }\end{array}$ & Asteraceae & $\mathrm{H}$ & D & Th & Mic & Dis & - & - & - & + \\
\hline
\end{tabular}


460 Muhammad Idrees, Wisal Muhammad Khan et al. Phytoclimatic Spectrum ....

\begin{tabular}{|c|c|c|c|c|c|c|c|c|c|c|c|}
\hline \multirow{2}{*}{$\begin{array}{l}\text { S. } \\
\text { No } \\
\end{array}$} & \multirow{2}{*}{ Species } & \multirow{2}{*}{ Family } & \multirow{2}{*}{$\begin{array}{l}\text { Ha } \\
\text { bit }\end{array}$} & \multirow{2}{*}{$\begin{array}{l}\text { Habit } \\
\text { at }\end{array}$} & \multirow{2}{*}{$\begin{array}{l}\text { Life } \\
\text { For } \\
\text { m }\end{array}$} & \multirow{2}{*}{$\begin{array}{c}\text { Leaf } \\
\text { Size } \\
\text { Spectru } \\
\text { m } \\
\end{array}$} & \multirow{2}{*}{$\begin{array}{c}\text { Lamin } \\
\text { a } \\
\text { Shape }\end{array}$} & \multicolumn{4}{|c|}{$\begin{array}{c}\text { Seasonalit } \\
y\end{array}$} \\
\hline & & & & & & & & A & $\mathbf{w}$ & $\begin{array}{l}\mathbf{S} \\
\mathbf{P}\end{array}$ & SM \\
\hline 76. & $\begin{array}{l}\text { Sonchus } \\
\text { arvensis L. }\end{array}$ & Asteraceae & $\mathrm{H}$ & D & Th & Mic & Dis & - & - & - & + \\
\hline 77. & $\begin{array}{l}\text { Seriphidium } \\
\text { brevifolium } \\
\text { (Wall. ex DC.) } \\
\text { Ling \& Y.R. Ling. }\end{array}$ & Asteraceae & $\mathrm{H}$ & $\mathrm{D}$ & $\mathrm{Np}$ & Mes & Dis & + & + & + & + \\
\hline 78. & $\begin{array}{l}\text { Silybum } \\
\text { marianum (L.) } \\
\text { Gaertn. }\end{array}$ & Asteraceae & $\mathrm{H}$ & WD & $\mathrm{Ch}$ & Mic & Sp & - & - & + & + \\
\hline 79. & $\begin{array}{l}\text { Taraxacum } \\
\text { officinale (L.) } \\
\text { Weber ex F.H. } \\
\text { Wigg. }\end{array}$ & Asteraceae & $\mathrm{H}$ & D & Th & Mic & $\mathrm{S}$ & + & - & + & + \\
\hline 80. & $\begin{array}{l}\text { Phagnalon } \\
\text { niveum Edgew. }\end{array}$ & Asteraceae & $\mathrm{H}$ & W & Ch & $\mathrm{N}$ & $S$ & + & - & + & + \\
\hline 81. & $\begin{array}{l}\text { Tagetes minuta } \\
\text { L. }\end{array}$ & Asteraceae & $\mathrm{H}$ & D & Th & $\mathrm{N}$ & Dis & - & - & - & + \\
\hline 82. & $\begin{array}{l}\text { Tragopogon } \\
\text { gracilis D. Don. }\end{array}$ & Asteraceae & $\mathrm{H}$ & D & Hem & Mic & $\mathrm{S}$ & - & - & + & + \\
\hline 83. & $\begin{array}{l}\text { Tussilago farfara } \\
\text { L. }\end{array}$ & Asteraceae & $\mathrm{H}$ & WD & G & Mes & $\mathrm{S}$ & - & - & + & + \\
\hline 84. & $\begin{array}{l}\text { Xanthium } \\
\text { strumarium L. }\end{array}$ & Asteraceae & $\mathrm{H}$ & WD & Th & Mes & $\mathrm{S}$ & - & - & - & + \\
\hline 85. & $\begin{array}{l}\text { Impatiens } \\
\text { edgeworthii } \\
\text { Hook. f. }\end{array}$ & Balsaminaceae & $\mathrm{H}$ & W & Th & Mic & $S$ & - & - & - & + \\
\hline 86. & $\begin{array}{l}\text { Berberis lycium } \\
\text { Royle. }\end{array}$ & Berberidaceae & $S$ & D & $\mathrm{Np}$ & $\mathrm{N}$ & Sp & + & + & + & + \\
\hline 87. & $\begin{array}{l}\text { Anchusa } \\
\text { arvensis (L.) } \\
\text { M.Bieb. }\end{array}$ & Boraginaceae & $\mathrm{H}$ & D & Th & Mic & S & - & - & + & \\
\hline 88. & $\begin{array}{l}\text { Arnebia } \\
\text { hispidissima } \\
\text { (Lehm.) A.D.C. }\end{array}$ & Boraginaceae & $\mathrm{H}$ & D & Th & $\mathrm{N}$ & $S$ & - & - & + & - \\
\hline 89. & $\begin{array}{l}\text { Cynoglossum } \\
\text { lanceolatum } \\
\text { Forssk. }\end{array}$ & Boraginaceae & $\mathrm{H}$ & D & Th & Mic & $S$ & - & + & + & - \\
\hline 90. & $\begin{array}{l}\text { Heliotropium } \\
\text { strigosum Willd. }\end{array}$ & Boraginaceae & $\mathrm{H}$ & WD & Th & Mic & $S$ & + & - & - & + \\
\hline 91. & $\begin{array}{l}\text { Heliotropium } \\
\text { europaeum L. }\end{array}$ & Boraginaceae & $\mathrm{H}$ & WD & Th & Mic & $S$ & - & - & + & - \\
\hline 92. & $\begin{array}{l}\text { Lithospermum } \\
\text { arvense L. }\end{array}$ & Boraginaceae & $\mathrm{H}$ & D & Th & $\mathrm{N}$ & $S$ & - & - & + & - \\
\hline 93. & $\begin{array}{l}\text { Myosotis } \\
\text { caespitosa } \\
\text { Schultz. }\end{array}$ & Boraginaceae & $\mathrm{H}$ & D & Hem & Mic & $S$ & - & - & - & + \\
\hline 94. & $\begin{array}{l}\text { Buddleja crispa } \\
\text { Benth. }\end{array}$ & Buddlejaceae & $S$ & D & $\mathrm{Np}$ & Mic & $S$ & + & + & + & + \\
\hline 95. & $\begin{array}{l}\text { Brassica } \\
\text { campestris L. }\end{array}$ & Brassicaceae & $\mathrm{H}$ & $\mathrm{CU}$ & Th & Mac & $S$ & - & + & + & - \\
\hline 96. & Brassica rapa L. & Brassicaceae & $\mathrm{H}$ & $\mathrm{CU}$ & Th & Mic & Dis & - & - & + & - \\
\hline 97. & Cardana draba & Brassicaceae & $\mathrm{H}$ & $\mathrm{D}$ & $\mathrm{Np}$ & Mic & $\mathrm{S}$ & - & \begin{tabular}{|l|}
- \\
\end{tabular} & + & - \\
\hline
\end{tabular}




\begin{tabular}{|c|c|c|c|c|c|c|c|c|c|c|c|}
\hline \multirow{2}{*}{$\begin{array}{l}\text { S. } \\
\text { No } \\
\text { - }\end{array}$} & \multirow{2}{*}{ Species } & \multirow{2}{*}{ Family } & \multirow{2}{*}{$\begin{array}{l}\text { Ha } \\
\text { bit }\end{array}$} & \multirow{2}{*}{$\begin{array}{c}\text { Habit } \\
\text { at }\end{array}$} & \multirow{2}{*}{$\begin{array}{c}\text { Life } \\
\text { For } \\
\text { m }\end{array}$} & \multirow{2}{*}{$\begin{array}{c}\text { Leaf } \\
\text { Size } \\
\text { Spectru } \\
\text { m }\end{array}$} & \multirow{2}{*}{$\begin{array}{c}\text { Lamin } \\
\text { a } \\
\text { Shape }\end{array}$} & \multicolumn{4}{|c|}{$\begin{array}{c}\text { Seasonalit } \\
y\end{array}$} \\
\hline & & & & & & & & $\mathbf{A}$ & $\mathbf{W}$ & $\begin{array}{l}\mathbf{S} \\
\mathbf{P}\end{array}$ & SM \\
\hline & (L.) Desv. & & & & & & & & & & \\
\hline 98. & $\begin{array}{l}\text { Coronopus } \\
\text { didymus (L.) } \\
\text { Sm. }\end{array}$ & Brassicaceae & $\mathrm{H}$ & W & Th & Mic & Dis & + & + & - & - \\
\hline 99. & Eruca sativa Mill. & Brassicaceae & $\mathrm{H}$ & W & $\mathrm{Np}$ & Mic & $\mathrm{S}$ & - & + & + & - \\
\hline 100 & $\begin{array}{l}\text { Lepidium } \\
\text { ruderale L. }\end{array}$ & Brassicaceae & $\mathrm{H}$ & $\mathrm{D}$ & Th & $\mathrm{N}$ & $S$ & - & - & - & + \\
\hline 101 & $\begin{array}{l}\text { Lepidium } \\
\text { didymum L. }\end{array}$ & Brassicaceae & $\mathrm{H}$ & WD & Hem & Lp & S & - & - & + & + \\
\hline 102 & $\begin{array}{l}\text { Nasturtium } \\
\text { officinale R.Br. }\end{array}$ & Brassicaceae & $\mathrm{H}$ & W & Th & $\mathrm{N}$ & S & - & - & + & - \\
\hline 103 & $\begin{array}{l}\text { Persicaria glabra } \\
\text { (Willd.) M. } \\
\text { Gomez. }\end{array}$ & Brassicaceae & $\mathrm{H}$ & W & $\mathrm{Np}$ & Mic & $S$ & - & - & + & + \\
\hline 104 & $\begin{array}{l}\text { Rorippa palustris } \\
\text { (L.) Besser. }\end{array}$ & Brassicaceae & $\mathrm{H}$ & $\mathrm{D}$ & Th & $\mathrm{N}$ & $S$ & - & - & + & - \\
\hline 105 & $\begin{array}{l}\text { Raphanus } \\
\text { sativus L. }\end{array}$ & Brassicaceae & $\mathrm{H}$ & W & Th & $\mathrm{N}$ & Comp & + & + & + & + \\
\hline 106 & $\begin{array}{l}\text { Sisymbrium irio } \\
\text { L. }\end{array}$ & Brassicaceae & $\mathrm{H}$ & $\mathrm{D}$ & Th & $\mathrm{N}$ & Dis & - & - & + & + \\
\hline 107 & $\begin{array}{l}\text { Thlaspi } \\
\text { perfoliatum L. }\end{array}$ & Brassicaceae & $\mathrm{H}$ & WD & Th & Mic & $S$ & - & + & + & + \\
\hline 108 & $\begin{array}{l}\text { Cannabis sativa } \\
\text { L. }\end{array}$ & Cannabaceae & $\mathrm{H}$ & WD & Th & Mic & S & - & - & + & + \\
\hline 109 & $\begin{array}{l}\text { Celtis caucasica } \\
\text { Willd. }\end{array}$ & Cannabaceae & $\mathrm{T}$ & $\mathrm{D}$ & Mic & Mic & S & + & + & + & + \\
\hline 110 & Canna indica L. & Cannaceae & $\mathrm{H}$ & WD & $\mathrm{Ch}$ & Meg & $\mathrm{S}$ & - & - & + & + \\
\hline 111 & $\begin{array}{l}\text { Arenaria } \\
\text { serpyllifolia L. }\end{array}$ & $\begin{array}{l}\text { Caryophyllace } \\
\text { ae }\end{array}$ & $\mathrm{H}$ & W & Th & Lp & $\mathrm{S}$ & + & - & - & + \\
\hline 112 & $\begin{array}{l}\text { Spergula } \\
\text { arvensis } \mathrm{L} .\end{array}$ & $\begin{array}{l}\text { Caryophyllace } \\
\text { ae }\end{array}$ & $\mathrm{H}$ & $\mathrm{D}$ & Hem & Lp & $S$ & - & - & - & + \\
\hline 113 & $\begin{array}{l}\text { Stellaria media } \\
\text { (L.) Vill. }\end{array}$ & $\begin{array}{l}\text { Caryophyllace } \\
\text { ae }\end{array}$ & $\mathrm{H}$ & W & $\mathrm{Ch}$ & $\mathrm{N}$ & Dis & - & - & + & + \\
\hline 114 & $\begin{array}{l}\text { Silene conoidea } \\
\text { L. }\end{array}$ & $\begin{array}{l}\text { Caryophyllace } \\
\text { ae }\end{array}$ & $\mathrm{H}$ & W & Th & Mic & $S$ & - & + & + & - \\
\hline 115 & $\begin{array}{l}\text { Silene viscosa } \\
\text { (L) Pers. }\end{array}$ & $\begin{array}{l}\text { Caryophyllace } \\
\text { ae }\end{array}$ & $\mathrm{H}$ & W & Th & Mic & $S$ & - & - & + & + \\
\hline 116 & $\begin{array}{l}\text { Opuntia dillenii } \\
\text { (Ker Gawl.) } \\
\text { Haw. }\end{array}$ & Cactaceae & $\mathrm{S}$ & $\mathrm{D}$ & $\mathrm{Np}$ & $\mathrm{N}$ & Abs & + & + & + & + \\
\hline 117 & $\begin{array}{l}\text { Convolvulus } \\
\text { arvensis L. }\end{array}$ & $\begin{array}{l}\text { Convolvulacea } \\
\text { e }\end{array}$ & $\mathrm{H}$ & WD & Th & $\mathrm{N}$ & $S$ & - & - & + & + \\
\hline 118 & $\begin{array}{l}\text { Cuscuta reflexa } \\
\text { Roxb. }\end{array}$ & Cuscutaceae & $\mathrm{H}$ & WD & $P$ & Lp & Abs & + & + & + & + \\
\hline 119 & $\begin{array}{l}\text { Chenopodium } \\
\text { album L. }\end{array}$ & $\begin{array}{l}\text { Chenopodiace } \\
\text { ae }\end{array}$ & $\mathrm{H}$ & WD & Th & $\mathrm{N}$ & $S$ & - & + & + & - \\
\hline 120 & $\begin{array}{l}\text { Chenopodium } \\
\text { ambrosioides L. }\end{array}$ & $\begin{array}{l}\text { Chenopodiace } \\
\text { ae }\end{array}$ & $\mathrm{H}$ & W & Th & Lp & $S$ & - & - & + & - \\
\hline 121 & $\begin{array}{l}\text { Chenopodium } \\
\text { murale L. }\end{array}$ & $\begin{array}{l}\text { Chenopodiace } \\
\text { ae }\end{array}$ & $\mathrm{H}$ & WD & Th & Lp & $S$ & + & - & - & - \\
\hline 122 & $\begin{array}{l}\text { Dysphania } \\
\text { botrys (L.) }\end{array}$ & $\begin{array}{l}\text { Chenopodiace } \\
\text { ae }\end{array}$ & $\mathrm{H}$ & WD & Th & Mic & $S$ & - & - & + & + \\
\hline
\end{tabular}


462 Muhammad Idrees, Wisal Muhammad Khan et al. Phytoclimatic Spectrum ....

\begin{tabular}{|c|c|c|c|c|c|c|c|c|c|c|c|}
\hline \multirow{2}{*}{$\begin{array}{l}\text { S. } \\
\text { No } \\
.\end{array}$} & \multirow{2}{*}{ Species } & \multirow{2}{*}{ Family } & \multirow{2}{*}{$\begin{array}{l}\text { Ha } \\
\text { bit }\end{array}$} & \multirow{2}{*}{$\begin{array}{c}\text { Habit } \\
\text { at }\end{array}$} & \multirow{2}{*}{$\begin{array}{l}\text { Life } \\
\text { For } \\
\text { m }\end{array}$} & \multirow{2}{*}{$\begin{array}{c}\text { Leaf } \\
\text { Size } \\
\text { Spectru } \\
\text { m }\end{array}$} & \multirow{2}{*}{$\begin{array}{c}\text { Lamin } \\
\text { a } \\
\text { Shape }\end{array}$} & \multicolumn{4}{|c|}{$\begin{array}{c}\text { Seasonalit } \\
y\end{array}$} \\
\hline & & & & & & & & A & $\mathbf{W}$ & $\begin{array}{l}\mathbf{S} \\
\mathbf{P}\end{array}$ & SM \\
\hline & $\begin{array}{l}\text { Mosyakin \& } \\
\text { Clemants. }\end{array}$ & & & & & & & & & & \\
\hline 123 & $\begin{array}{l}\text { Spinacia } \\
\text { oleracea L. }\end{array}$ & $\begin{array}{l}\text { Chenopodiace } \\
\text { ae }\end{array}$ & $\mathrm{H}$ & $\mathrm{CU}$ & Th & Mic & $S$ & - & + & + & - \\
\hline 124 & $\begin{array}{l}\text { Cucumis sativus } \\
\text { L. }\end{array}$ & Cucurbitaceae & $\mathrm{H}$ & $\mathrm{CU}$ & Th & Mac & Dis & + & - & + & - \\
\hline 125 & Cucumis melo L. & Cucurbitaceae & $\mathrm{H}$ & $\mathrm{CU}$ & Th & Mic & $\mathrm{S}$ & + & - & + & - \\
\hline 126 & $\begin{array}{l}\text { Cucurbita pepo } \\
\text { L. }\end{array}$ & Cucurbitaceae & $\mathrm{H}$ & $\mathrm{CU}$ & Th & Meg & Dis & + & - & + & - \\
\hline 127 & $\begin{array}{l}\text { Cucurbita } \\
\text { maxima } \\
\text { Duchesne. }\end{array}$ & Cucurbitaceae & $\mathrm{H}$ & $\mathrm{CU}$ & Th & Mac & Dis & - & - & + & - \\
\hline 128 & $\begin{array}{l}\text { Luffa cylindrica } \\
\text { (L.) M.Roem. }\end{array}$ & Cucurbitaceae & $\mathrm{H}$ & $\mathrm{CU}$ & Th & Mac & Dis & - & + & + & - \\
\hline 129 & $\begin{array}{l}\text { Momordica } \\
\text { charantia L. }\end{array}$ & Cucurbitaceae & $\mathrm{H}$ & $\mathrm{CU}$ & Th & Mes & Dis & + & - & + & - \\
\hline 130 & $\begin{array}{l}\text { Diospyros kaki } \\
\text { L.f. }\end{array}$ & Ebenaceae & $\mathrm{T}$ & $\mathrm{D}$ & Mes & Mes & $\mathrm{S}$ & + & + & + & + \\
\hline 131 & $\begin{array}{l}\text { Diospyros lotus } \\
\text { L. }\end{array}$ & Ebenaceae & $\mathrm{T}$ & $\mathrm{D}$ & Meg & Mic & S & + & + & + & + \\
\hline 132 & $\begin{array}{l}\text { Euphorbia } \\
\text { granulata } \\
\text { Forssk. }\end{array}$ & Euphorbiaceae & $\mathrm{H}$ & $\mathrm{D}$ & Hem & Lp & $\mathrm{S}$ & + & - & - & - \\
\hline 133 & $\begin{array}{l}\text { Euphorbia } \\
\text { helioscopia L. }\end{array}$ & Euphorbiaceae & $\mathrm{H}$ & $\mathrm{D}$ & Th & $\mathrm{N}$ & $\mathrm{S}$ & - & - & + & - \\
\hline 134 & $\begin{array}{l}\text { Euphorbia } \\
\text { prostrata Ait }\end{array}$ & Euphorbiaceae & $\mathrm{H}$ & $\mathrm{D}$ & Th & $\mathrm{N}$ & $\mathrm{S}$ & - & - & + & - \\
\hline 135 & $\begin{array}{l}\text { Euphorbia } \\
\text { heterophylla L. }\end{array}$ & Euphorbiaceae & $\mathrm{H}$ & D & Th & Mic & $\mathrm{S}$ & - & - & + & + \\
\hline 136 & $\begin{array}{l}\text { Euphorbia hirta } \\
\text { L. }\end{array}$ & Euphorbiaceae & $\mathrm{H}$ & D & $\mathrm{Ch}$ & $\mathrm{N}$ & $\mathrm{S}$ & - & - & + & + \\
\hline 137 & $\begin{array}{l}\text { Euphorbia } \\
\text { serrata L. }\end{array}$ & Euphorbiaceae & $\mathrm{H}$ & $\mathrm{D}$ & Th & $\mathrm{N}$ & $\mathrm{S}$ & - & - & + & - \\
\hline 138 & $\begin{array}{l}\text { Ricinus } \\
\text { communis L. }\end{array}$ & Euphorbiaceae & $\mathrm{S}$ & WD & $\mathrm{Np}$ & Mac & $S$ & + & + & + & + \\
\hline 139 & $\begin{array}{l}\text { Quercus baloot } \\
\text { Griff. }\end{array}$ & Fagaceae & $\mathrm{T}$ & $\mathrm{D}$ & Mes & Mic & $S$ & + & + & + & + \\
\hline 140 & $\begin{array}{l}\text { Quercus incana } \\
\text { Bartram. }\end{array}$ & Fagaceae & $\mathrm{T}$ & $\mathrm{D}$ & Mes & Mic & $\mathrm{S}$ & + & + & + & + \\
\hline 141 & $\begin{array}{l}\text { Quercus dilatata } \\
\text { Royle. }\end{array}$ & Fagaceae & $\mathrm{T}$ & $\mathrm{D}$ & Mes & Mic & $\mathrm{S}$ & + & + & + & + \\
\hline 142 & $\begin{array}{l}\text { Fumaria indica } \\
\text { (Hausskn.) } \\
\text { Pugsley. }\end{array}$ & Fumariaceae & $\mathrm{H}$ & WD & Th & $\mathrm{N}$ & Dis & - & - & + & + \\
\hline 143 & Vicia sativa L. & Fum & $\mathrm{H}$ & D & Th & $\mathrm{N}$ & Comp & - & - & + & + \\
\hline 144 & Juglans regia L. & Juglandaceae & $\mathrm{T}$ & $\mathrm{D}$ & Mes & Mic & Comp & + & + & + & + \\
\hline 145 & $\begin{array}{l}\text { Ajuga bracteosa } \\
\text { Wall. ex Benth. }\end{array}$ & Lamiaceae & $\mathrm{H}$ & D & Hem & Mic & $\mathrm{S}$ & + & - & + & + \\
\hline 146 & $\begin{array}{l}\text { Ajuga parviflora } \\
\text { Benth. }\end{array}$ & Lamiaceae & $\mathrm{H}$ & $\mathrm{D}$ & Th & Mes & S & + & + & - & + \\
\hline 147 & $\begin{array}{l}\text { Mentha arvensis } \\
\text { L. }\end{array}$ & Lamiaceae & $\mathrm{H}$ & W & G & $\mathrm{N}$ & $\mathrm{S}$ & + & - & + & + \\
\hline
\end{tabular}




\begin{tabular}{|c|c|c|c|c|c|c|c|c|c|c|c|}
\hline \multirow{2}{*}{$\begin{array}{l}\text { S. } \\
\text { No } \\
\text { - }\end{array}$} & \multirow{2}{*}{ Species } & \multirow{2}{*}{ Family } & \multirow{2}{*}{$\begin{array}{l}\text { Ha } \\
\text { bit }\end{array}$} & \multirow{2}{*}{$\begin{array}{c}\text { Habit } \\
\text { at }\end{array}$} & \multirow{2}{*}{$\begin{array}{l}\text { Life } \\
\text { For } \\
\text { m }\end{array}$} & \multirow{2}{*}{$\begin{array}{c}\text { Leaf } \\
\text { Size } \\
\text { Spectru } \\
\text { m }\end{array}$} & \multirow{2}{*}{$\begin{array}{c}\text { Lamin } \\
\text { a } \\
\text { Shape }\end{array}$} & \multicolumn{4}{|c|}{$\begin{array}{c}\text { Seasonalit } \\
y\end{array}$} \\
\hline & & & & & & & & A & $\mathbf{w}$ & $\begin{array}{l}\mathbf{S} \\
\mathbf{P}\end{array}$ & SM \\
\hline 148 & $\begin{array}{l}\text { Mentha longifolia } \\
\text { L. }\end{array}$ & Lamiaceae & $\mathrm{H}$ & W & Hem & $\mathrm{N}$ & S & + & - & + & + \\
\hline 149 & $\begin{array}{l}\text { Ocimum } \\
\text { bascillicum L. }\end{array}$ & Lamiaceae & $S$ & $\mathrm{D}$ & $\mathrm{Ch}$ & $\mathrm{N}$ & $S$ & + & + & + & + \\
\hline 150 & $\begin{array}{l}\text { Salvia lanata } \\
\text { Roxb. }\end{array}$ & Lamiaceae & $\mathrm{H}$ & $\mathrm{D}$ & Th & Mes & $S$ & + & + & - & + \\
\hline 151 & $\begin{array}{l}\text { Salvia } \\
\text { moorcroftiana } \\
\text { Wall. ex Benth. }\end{array}$ & Lamiaceae & $\mathrm{H}$ & $\mathrm{D}$ & Th & Mac & $S$ & - & - & + & + \\
\hline 152 & $\begin{array}{l}\text { Salvia nubicola } \\
\text { Wall. ex Sweet. }\end{array}$ & Lamiaceae & $\mathrm{H}$ & $\mathrm{D}$ & Th & Mes & $\mathrm{S}$ & + & + & - & + \\
\hline 153 & $\begin{array}{l}\text { Punica granatum } \\
\text { L. }\end{array}$ & Lytheraceae & $\mathrm{T}$ & $\mathrm{D}$ & Mes & $\mathrm{N}$ & $\mathrm{S}$ & + & + & + & + \\
\hline 154 & $\begin{array}{l}\text { Abelmoschus } \\
\text { esculentus (L.) } \\
\text { Moench. }\end{array}$ & Malvaceae & $\mathrm{H}$ & $\mathrm{CU}$ & Th & Mes & Sp & + & - & - & + \\
\hline 155 & $\begin{array}{l}\text { Malva neglecta } \\
\text { Wallr. }\end{array}$ & Malvaceae & $\mathrm{H}$ & $D$ & Th & Mic & $S$ & - & + & + & - \\
\hline 156 & $\begin{array}{l}\text { Melia azedarach } \\
\text { L. }\end{array}$ & Meliaceae & $\mathrm{T}$ & $\mathrm{D}$ & Meg & Mic & Comp & + & + & + & + \\
\hline 157 & $\begin{array}{l}\text { Acacia modesta } \\
\text { Wall. }\end{array}$ & Mimosaceae & $\mathrm{T}$ & $D$ & Mic & Lp & Comp & + & + & + & + \\
\hline 158 & $\begin{array}{l}\text { Acacia nilotica } \\
\text { (L.) Delile. }\end{array}$ & Mimosaceae & $\mathrm{T}$ & $\mathrm{D}$ & MIic & Lp & Comp & + & + & + & + \\
\hline 159 & $\begin{array}{l}\text { Broussonetia } \\
\text { papyrifera L. }\end{array}$ & Moraceae & $\mathrm{T}$ & WD & Meg & Mes & $\mathrm{S}$ & + & + & + & + \\
\hline 160 & Ficus carica L. & Moraceae & $\mathrm{T}$ & $\mathrm{D}$ & Meg & Mes & $\mathrm{S}$ & + & + & + & + \\
\hline 161 & $\begin{array}{l}\text { Ficus palmata } \\
\text { Forssk. }\end{array}$ & Moraceae & $\mathrm{T}$ & $\mathrm{D}$ & Meg & Mes & S & + & + & + & + \\
\hline 162 & $\begin{array}{l}\text { Ficus } \\
\text { sarmentosa } \\
\text { Buch. -Ham. Ex } \\
\text { Sm. }\end{array}$ & Moraceae & $\mathrm{T}$ & $\mathrm{D}$ & Meg & Mes & $S$ & + & + & + & + \\
\hline 163 & Morus alba L. & Moraceae & $\mathrm{T}$ & $\mathrm{D}$ & Meg & Mes & $\mathrm{S}$ & + & + & + & + \\
\hline 164 & $\begin{array}{l}\text { Morus macroura } \\
\text { Miq. }\end{array}$ & Moraceae & $\mathrm{T}$ & D & Meg & Mes & $S$ & + & + & + & + \\
\hline 165 & Morus nigra L. & Moraceae & $\mathrm{T}$ & $\mathrm{D}$ & Meg & Mes & $\mathrm{S}$ & + & + & + & + \\
\hline 166 & $\begin{array}{l}\text { Psidium guajava } \\
\text { L. }\end{array}$ & Myrtaceae & $\mathrm{T}$ & $\mathrm{D}$ & Mes & Mes & $\mathrm{S}$ & + & + & + & + \\
\hline 167 & $\begin{array}{l}\text { Eucalyptus } \\
\text { camaldulensis } \\
\text { Dehnh. }\end{array}$ & Myrtaceae & $\mathrm{T}$ & $\mathrm{D}$ & Meg & $\mathrm{N}$ & $S$ & + & + & + & + \\
\hline 168 & $\begin{array}{l}\text { Mirabilis jalapa } \\
\text { L. }\end{array}$ & Nyctaginaceae & $\mathrm{H}$ & $\mathrm{D}$ & $\mathrm{Np}$ & Mes & $\mathrm{S}$ & + & - & + & + \\
\hline 169 & $\begin{array}{l}\text { Jasminum } \\
\text { officinale L. }\end{array}$ & Oleaceae & $\mathrm{H}$ & $\mathrm{D}$ & $\mathrm{Np}$ & Mic & Comp & + & + & + & + \\
\hline 170 & $\begin{array}{l}\text { Jasminum } \\
\text { humile L. }\end{array}$ & Oleaceae & $\mathrm{H}$ & WD & $\mathrm{Np}$ & Mic & Comp & + & + & + & + \\
\hline 171 & $\begin{array}{l}\text { Olea ferruginea } \\
\text { Wall. ex Aitch. }\end{array}$ & Oleaceae & $\mathrm{T}$ & $\mathrm{D}$ & Mes & Mic & $S$ & + & + & + & + \\
\hline 172 & $\begin{array}{l}\text { Papaver } \\
\text { somniferum L. }\end{array}$ & Papaveraceae & $\mathrm{H}$ & D & Th & Mes & Dis & - & - & + & + \\
\hline
\end{tabular}


464 Muhammad Idrees, Wisal Muhammad Khan et al. Phytoclimatic Spectrum ....

\begin{tabular}{|c|c|c|c|c|c|c|c|c|c|c|c|}
\hline \multirow{2}{*}{$\begin{array}{l}\text { S. } \\
\text { No }\end{array}$} & \multirow{2}{*}{ Species } & \multirow{2}{*}{ Family } & \multirow{2}{*}{$\begin{array}{l}\text { Ha } \\
\text { bit }\end{array}$} & \multirow{2}{*}{$\begin{array}{c}\text { Habit } \\
\text { at }\end{array}$} & \multirow{2}{*}{$\begin{array}{l}\text { Life } \\
\text { For } \\
\text { m }\end{array}$} & \multirow{2}{*}{$\begin{array}{c}\text { Leaf } \\
\text { Size } \\
\text { Spectru } \\
\text { m }\end{array}$} & \multirow{2}{*}{$\begin{array}{l}\text { Lamin } \\
\text { a } \\
\text { Shape }\end{array}$} & \multicolumn{4}{|c|}{$\begin{array}{c}\text { Seasonalit } \\
y\end{array}$} \\
\hline & & & & & & & & A & $\mathbf{W}$ & $\begin{array}{l}\mathbf{S} \\
\mathbf{P}\end{array}$ & SM \\
\hline 173 & $\begin{array}{l}\text { Lathyrus aphaca } \\
\text { L. }\end{array}$ & Papilonaceae & $\mathrm{H}$ & WD & Th & Mic & Comp & - & - & + & - \\
\hline 174 & $\begin{array}{l}\text { Medicago } \\
\text { denticulata } \\
\text { Willd. }\end{array}$ & Papilonaceae & $\mathrm{H}$ & WD & Th & $\mathrm{N}$ & Comp & - & - & + & + \\
\hline 175 & $\begin{array}{l}\text { Medicago } \\
\text { polymorpha L. }\end{array}$ & Papilonaceae & $\mathrm{H}$ & WD & Th & $\mathrm{N}$ & Comp & - & + & + & - \\
\hline 176 & $\begin{array}{l}\text { Medicago } \\
\text { minima L. }\end{array}$ & Papilonaceae & $\mathrm{H}$ & WD & Th & $\mathrm{N}$ & Comp & - & + & + & - \\
\hline 177 & $\begin{array}{l}\text { Medicago } \\
\text { Iupulina L. }\end{array}$ & Papilonaceae & $\mathrm{H}$ & D & Th & $\mathrm{N}$ & Comp & - & - & + & + \\
\hline 178 & $\begin{array}{l}\text { Phaseolus } \\
\text { vulgaris L. }\end{array}$ & Papilonaceae & $\mathrm{H}$ & $\mathrm{CU}$ & Ch & Mes & Comp & - & - & - & + \\
\hline 179 & $\begin{array}{l}\text { Phaseolus } \\
\text { lunatus L. }\end{array}$ & Papilonaceae & $\mathrm{H}$ & $\mathrm{CU}$ & $\mathrm{Ch}$ & Mes & Comp & - & - & + & - \\
\hline 180 & Pisum sativum L. & Papilonaceae & $\mathrm{H}$ & $\mathrm{CU}$ & Th & Mic & Comp & $\begin{array}{l}- \\
+\end{array}$ & - & + & - \\
\hline 181 & $\begin{array}{l}\text { Trifolium repens } \\
\text { L. }\end{array}$ & Papilonaceae & $\mathrm{H}$ & WD & Hem & $\mathrm{N}$ & Comp & - & - & + & + \\
\hline 182 & $\begin{array}{l}\text { Trifolium } \\
\text { resupinatum L. }\end{array}$ & Papilonaceae & $\mathrm{H}$ & $\mathrm{CU}$ & Hem & Mic & Comp & - & - & + & + \\
\hline 183 & $\begin{array}{l}\text { Pinus roxburghii } \\
\text { Sarg. }\end{array}$ & Pinaceae & $\mathrm{T}$ & $\mathrm{D}$ & Mes & Lp & $\mathrm{N}$ & + & + & + & + \\
\hline 184 & $\begin{array}{l}\text { Pinus wallichiana } \\
\text { A.B. Jacks. }\end{array}$ & Pinaceae & $\mathrm{T}$ & $\mathrm{D}$ & Meg & Lp & $\mathrm{N}$ & + & + & + & + \\
\hline 185 & $\begin{array}{l}\text { Platanus } \\
\text { orientalis L. }\end{array}$ & Platanaceae & $\mathrm{T}$ & WD & Meg & Mes & Dis & + & + & + & + \\
\hline 186 & $\begin{array}{l}\text { Rumex dentatus } \\
\text { L. }\end{array}$ & Polygonaceae & $\mathrm{H}$ & W & Ch & Mes & $\mathrm{S}$ & + & - & - & + \\
\hline 187 & $\begin{array}{l}\text { Rumex hastatus } \\
\text { D. Don. }\end{array}$ & Polygonaceae & $\mathrm{H}$ & W & $\mathrm{Ch}$ & $\mathrm{N}$ & Dis & + & + & - & + \\
\hline 188 & $\begin{array}{l}\text { Ranunculus } \\
\text { arvensis L. }\end{array}$ & Rananculaceae & $\mathrm{H}$ & WD & G & $\mathrm{N}$ & Dis & - & - & + & - \\
\hline 189 & $\begin{array}{l}\text { Ranunculus } \\
\text { muricatus L. }\end{array}$ & Rananculaceae & $\mathrm{H}$ & WD & G & $\mathrm{N}$ & Dis & - & - & + & + \\
\hline 190 & $\begin{array}{l}\text { Ranunculus } \\
\text { sceleratus L. }\end{array}$ & Rananculaceae & $\mathrm{H}$ & WD & G & $\mathrm{N}$ & Dis & - & - & + & + \\
\hline 191 & $\begin{array}{l}\text { Ziziphus sativa } \\
\text { Gaertn. }\end{array}$ & Rhamnaceae & $\mathrm{T}$ & $\mathrm{D}$ & Meg & $\mathrm{N}$ & $S$ & + & + & + & + \\
\hline 192 & $\begin{array}{l}\text { Ziziphus jujuba } \\
\text { Mill. }\end{array}$ & Rhamnaceae & $\mathrm{T}$ & D & Mes & Mic & $\mathrm{S}$ & + & + & + & + \\
\hline 193 & $\begin{array}{l}\text { Ziziphus } \\
\text { oxyphylla } \\
\text { Edgew. }\end{array}$ & Rhamnaceae & $\mathrm{T}$ & $\mathrm{D}$ & $\mathrm{Np}$ & $\mathrm{N}$ & $S$ & + & + & + & + \\
\hline 194 & $\begin{array}{l}\text { Cotoneaster } \\
\text { microphyllus } \\
\text { Wall. ex Lindl. }\end{array}$ & Rosaceae & $\mathrm{H}$ & WD & $\mathrm{Np}$ & Lp & S & + & + & + & + \\
\hline 195 & $\begin{array}{l}\text { Cotoneaster } \\
\text { nummularia } \\
\text { Fisch \& Mey. }\end{array}$ & Rosaceae & $\mathrm{H}$ & WD & $\mathrm{Np}$ & $\mathrm{N}$ & $S$ & + & + & + & + \\
\hline 196 & $\begin{array}{l}\text { Eriobotrys } \\
\text { japonica }\end{array}$ & Rosaceae & $\mathrm{T}$ & D & Mic & Mes & S & + & + & + & + \\
\hline
\end{tabular}




\begin{tabular}{|c|c|c|c|c|c|c|c|c|c|c|c|}
\hline \multirow{2}{*}{$\begin{array}{l}\text { S. } \\
\text { No } \\
\end{array}$} & \multirow{2}{*}{ Species } & \multirow{2}{*}{ Family } & \multirow{2}{*}{$\begin{array}{l}\text { Ha } \\
\text { bit }\end{array}$} & \multirow{2}{*}{$\begin{array}{l}\text { Habit } \\
\text { at }\end{array}$} & \multirow{2}{*}{$\begin{array}{c}\text { Life } \\
\text { For } \\
\text { m }\end{array}$} & \multirow{2}{*}{$\begin{array}{c}\text { Leaf } \\
\text { Size } \\
\text { Spectru } \\
\text { m }\end{array}$} & \multirow{2}{*}{$\begin{array}{c}\text { Lamin } \\
\text { a } \\
\text { Shape }\end{array}$} & \multicolumn{4}{|c|}{\begin{tabular}{|c} 
Seasonalit \\
$y$ \\
\end{tabular}} \\
\hline & & & & & & & & $\mathbf{A}$ & $\mathbf{W}$ & $\begin{array}{l}\mathbf{S} \\
\mathbf{P}\end{array}$ & SM \\
\hline & (Thunb.) Lindl. & & & & & & & & & & \\
\hline 197 & $\begin{array}{l}\text { Prunus persica } \\
\text { (L.) Batsch. }\end{array}$ & Rosaceae & $\mathrm{T}$ & $\mathrm{CU}$ & Mic & Mes & S & + & + & + & + \\
\hline 198 & $\begin{array}{l}\text { Prunus } \\
\text { domestica L. }\end{array}$ & Rosaceae & $\mathrm{T}$ & $\mathrm{CU}$ & Mic & Mes & $\mathrm{S}$ & + & + & + & + \\
\hline 199 & $\begin{array}{l}\text { Prunus } \\
\text { armeniaca L. }\end{array}$ & Rosaceae & $\mathrm{T}$ & $\mathrm{CU}$ & Mic & Mes & $\mathrm{S}$ & + & + & + & + \\
\hline 200 & Pyrus baccata L. & Rosaceae & $\mathrm{T}$ & WD & Mic & Mes & $S$ & + & + & + & + \\
\hline 201 & $\begin{array}{l}\text { Pyrus communis } \\
\text { L. }\end{array}$ & Rosaceae & $\mathrm{T}$ & $\mathrm{CU}$ & Mic & Mes & $\mathrm{S}$ & + & + & + & + \\
\hline 202 & Pyrus malus L. & Rosaceae & $\mathrm{T}$ & $\mathrm{CU}$ & Mic & Mes & $\mathrm{S}$ & + & + & + & + \\
\hline 203 & $\begin{array}{l}\text { Pyrus pashia } \\
\text { Buch. -Ham. ex } \\
\text { D. Don. }\end{array}$ & Rosaceae & $\mathrm{T}$ & $\mathrm{CU}$ & Mic & Mes & $S$ & + & + & + & + \\
\hline 204 & Rosa alba L. & Rosaceae & $S$ & $E$ & $\mathrm{~Np}$ & $\mathrm{~N}$ & Comp & + & + & + & + \\
\hline 205 & $\begin{array}{l}\text { Rosa webbiana } \\
\text { Wall. ex Royle. }\end{array}$ & Rosaceae & $\mathrm{S}$ & WD & $\mathrm{Np}$ & $\mathrm{N}$ & Comp & + & + & + & + \\
\hline 206 & Rosa indica L. & Rosaceae & $\mathrm{S}$ & $\mathrm{CU}$ & $\mathrm{Np}$ & $\mathrm{N}$ & Comp & + & + & + & + \\
\hline 207 & $\begin{array}{l}\text { Rubus ellipticus } \\
\text { Sm. }\end{array}$ & Rosaceae & $\mathrm{S}$ & D & $\mathrm{Np}$ & $\mathrm{N}$ & Comp & + & + & + & + \\
\hline 208 & $\begin{array}{l}\text { Rubus fruticosus } \\
\text { L. }\end{array}$ & Rosaceae & $\mathrm{S}$ & $\mathrm{D}$ & Mes & $\mathrm{N}$ & Comp & + & + & + & + \\
\hline 209 & $\begin{array}{l}\text { Rubus ulmifolius } \\
\text { Schott. }\end{array}$ & Rosaceae & $\mathrm{S}$ & $\mathrm{D}$ & Mes & $\mathrm{N}$ & Comp & + & + & + & + \\
\hline 210 & $\begin{array}{l}\text { Citrus indica Yu. } \\
\text { Tanaka. }\end{array}$ & Rotaceae & $\mathrm{S}$ & $\mathrm{CU}$ & Mic & Mes & S & + & + & + & + \\
\hline 211 & $\begin{array}{l}\text { Citrus limetta } \\
\text { Risso. }\end{array}$ & Rotaceae & $\mathrm{S}$ & $\mathrm{CU}$ & Mic & Mes & $S$ & + & + & + & + \\
\hline 212 & $\begin{array}{l}\text { Citrus sinensis } \\
\text { (L.) Osbeck. }\end{array}$ & Rotaceae & $\mathrm{S}$ & $\mathrm{CU}$ & Mic & Mes & $\mathrm{S}$ & + & + & + & + \\
\hline 213 & $\begin{array}{l}\text { Salix } \\
\text { tetrasperma } \\
\text { Roxb. }\end{array}$ & Salicaceae & $\mathrm{T}$ & W & Mic & Mic & $\mathrm{S}$ & + & + & + & + \\
\hline 214 & Salix alba L. & Salicaceae & $\mathrm{T}$ & W & Meg & $M$ & $\mathrm{~S}$ & + & + & + & + \\
\hline 215 & Populus alba L. & Salic & $\mathrm{T}$ & $v$ & Mes & Mes & $s$ & + & + & + & + \\
\hline 216 & Populus nigra L. & Salic & $\mathrm{T}$ & 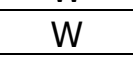 & Meg & Mic & $s$ & + & + & + & + \\
\hline 217 & $\begin{array}{l}\text { Dodonaea } \\
\text { viscosa (L.) } \\
\text { Jacq. }\end{array}$ & Sapindaceae & $\mathrm{S}$ & $\mathrm{D}$ & $\mathrm{Np}$ & $\mathrm{N}$ & $\mathrm{S}$ & + & + & + & + \\
\hline 218 & $\begin{array}{l}\text { Ailanthus } \\
\text { altissima } \\
\text { (Mill.)Swingle. }\end{array}$ & $\begin{array}{l}\text { Simaroubacea } \\
\text { e }\end{array}$ & $\mathrm{T}$ & $\mathrm{D}$ & Mic & Mic & Comp & + & + & + & + \\
\hline 219 & $\begin{array}{l}\text { Atropa } \\
\text { acuminate } \\
\text { Royal. ex Lindl. }\end{array}$ & Solanaceae & $\mathrm{H}$ & $\mathrm{D}$ & Th & Mes & $S$ & - & - & - & + \\
\hline 220 & $\begin{array}{l}\text { Capsicum } \\
\text { annuum L. }\end{array}$ & Solanaceae & $\mathrm{S}$ & $\mathrm{CU}$ & Th & Mic & S & + & - & - & + \\
\hline 221 & $\begin{array}{l}\text { Capsicum } \\
\text { frutescens L. }\end{array}$ & Solanaceae & $\mathrm{S}$ & $\mathrm{CU}$ & $\mathrm{Np}$ & Mes & $S$ & + & + & + & + \\
\hline 222 & $\begin{array}{l}\text { Cestrum } \\
\text { nocturnum L. }\end{array}$ & Solanaceae & $\mathrm{S}$ & D & $\mathrm{Ch}$ & Mes & $\mathrm{S}$ & + & + & + & + \\
\hline 223 & Datura innoxia & Solanaceae & $\mathrm{H}$ & $\mathrm{D}$ & Th & Mes & $\mathrm{S}$ & + & - & - & + \\
\hline
\end{tabular}


466 Muhammad Idrees, Wisal Muhammad Khan et al. Phytoclimatic Spectrum ....

\begin{tabular}{|c|c|c|c|c|c|c|c|c|c|c|c|}
\hline \multirow{2}{*}{$\begin{array}{l}\text { S. } \\
\text { No } \\
.\end{array}$} & \multirow{2}{*}{ Species } & \multirow{2}{*}{ Family } & \multirow{2}{*}{$\begin{array}{l}\text { Ha } \\
\text { bit }\end{array}$} & \multirow{2}{*}{$\begin{array}{c}\text { Habit } \\
\text { at }\end{array}$} & \multirow{2}{*}{$\begin{array}{c}\text { Life } \\
\text { For } \\
\text { m }\end{array}$} & \multirow{2}{*}{$\begin{array}{c}\text { Leaf } \\
\text { Size } \\
\text { Spectru } \\
\text { m }\end{array}$} & \multirow{2}{*}{$\begin{array}{c}\text { Lamin } \\
\text { a } \\
\text { Shape }\end{array}$} & \multicolumn{4}{|c|}{$\begin{array}{c}\text { Seasonalit } \\
y\end{array}$} \\
\hline & & & & & & & & A & $\mathbf{w}$ & $\begin{array}{l}\mathbf{S} \\
\mathbf{P}\end{array}$ & SM \\
\hline & Mill. & & & & & & & & & & \\
\hline 224 & $\begin{array}{l}\text { Datura } \\
\text { stramonium L. }\end{array}$ & Solanaceae & $\mathrm{H}$ & D & Th & Mes & $\mathrm{S}$ & - & - & - & + \\
\hline 225 & $\begin{array}{l}\text { Solanum nigrum } \\
\text { L. }\end{array}$ & Solanaceae & $\mathrm{H}$ & $\mathrm{CU}$ & Th & Mes & $\mathrm{S}$ & + & - & - & + \\
\hline 226 & $\begin{array}{l}\text { Solanum } \\
\text { tuberosum L. }\end{array}$ & Solanaceae & $\mathrm{H}$ & $\mathrm{CU}$ & G & Mes & Comp & - & - & - & + \\
\hline 227 & $\begin{array}{l}\text { Solanum } \\
\text { surattense } \\
\text { Burm. F. }\end{array}$ & Solanaceae & $\mathrm{H}$ & $\mathrm{CU}$ & Hem & Mes & $S$ & - & - & - & + \\
\hline 228 & $\begin{array}{l}\text { Withania } \\
\text { somnifera (L.) } \\
\text { Dunal. }\end{array}$ & Solanaceae & $\mathrm{S}$ & D & Ch & Mic & $S$ & + & + & + & + \\
\hline 229 & $\begin{array}{l}\text { Withania } \\
\text { coagulans } \\
\text { (Stocks) Dunal. }\end{array}$ & Solanaceae & $\mathrm{S}$ & D & $\mathrm{Ch}$ & Mic & $S$ & + & + & + & + \\
\hline 230 & Celtis australis L. & Ulmaceae & $\mathrm{T}$ & $\mathrm{D}$ & Meg & Mic & $\mathrm{S}$ & + & + & + & + \\
\hline 231 & $\begin{array}{l}\text { Celtis caucasica } \\
\text { Willd. }\end{array}$ & Ulmaceae & $\mathrm{T}$ & D & Mes & Mic & $\mathrm{S}$ & + & + & + & + \\
\hline 232 & Urtica dioica L. & Urticaceae & $\mathrm{H}$ & W & Th & Mic & $\mathrm{S}$ & - & - & + & + \\
\hline 233 & Viola biflora L. & Urticaceae & $\mathrm{H}$ & $\mathrm{W}$ & Th & Mic & $\mathrm{S}$ & - & - & + & + \\
\hline 234 & $\begin{array}{l}\text { Viola canescens } \\
\text { Wall. }\end{array}$ & Urticaceae & $\mathrm{H}$ & D & Th & Mic & $S$ & - & - & + & - \\
\hline 235 & Vitis vinifera L. & Vitaceae & $\mathrm{S}$ & $\mathrm{CU}$ & $\mathrm{Np}$ & Mes & $\mathrm{S}$ & + & + & + & + \\
\hline 236 & $\begin{array}{l}\text { Aloe vera (L.) } \\
\text { Burm.f. }\end{array}$ & $\begin{array}{l}\text { Xanthorrhoerc } \\
\text { eae }\end{array}$ & $\mathrm{H}$ & D & $\mathrm{Ch}$ & Mes & $S$ & + & + & + & + \\
\hline 237 & $\begin{array}{l}\text { Tribulus } \\
\text { terrestris L. }\end{array}$ & Zygophyllacae & $\mathrm{H}$ & D & Hem & Lp & Comp & + & - & - & + \\
\hline 238 & $\begin{array}{l}\text { Peganum } \\
\text { harmala L. }\end{array}$ & Zygophyllacae & $\mathrm{H}$ & D & Th & Lp & Dis & - & - & + & - \\
\hline
\end{tabular}

Table 4: Key to abbreviations

\begin{tabular}{|l|l|l|l|l|}
\hline Habit & Habitat & Life form classes & $\begin{array}{l}\text { Leaf size } \\
\text { spectrum }\end{array}$ & Lamina shape \\
\hline $\mathrm{H}=$ Herb & $\mathrm{D}=$ Dry & Hem=Hemicryptiphytes & Mac=Macrophyll & S=Simple \\
\hline $\begin{array}{l}\mathrm{S}= \\
\text { Shrub }\end{array}$ & W= Wet & Mic=Microphanerophyte & Mic =Microphyll & Dis =Dissected \\
\hline $\mathrm{T}=$ Tree & $\mathrm{CU}=$ Cultivated & Mes=Mesophanerophyte & Mes=Mesophyll & $\begin{array}{l}\text { Comp } \\
\text { =Compound }\end{array}$ \\
\hline & WD=Dry/Wet & Meg=Megaphanerophyte & Meg=Megaphyll & Abs=Abesent \\
\hline & $\mathrm{G}=$ Geophtes & Th=Therophytes & Lp =Leptophyll & $\mathrm{N}=$ Needle \\
\hline & $\mathrm{P}=$ Parasite & $\mathrm{Ch}=$ Chamaephytes & $\mathrm{N}=$ Nanophyll & $\mathrm{Sp}=$ Spiny \\
\hline & & $\mathrm{Np}=$ Nanophanerophyte & $\mathrm{Ap}=$ Aphyllous & \\
\hline
\end{tabular}


Table 5: Biological characteristics of the Valley

\begin{tabular}{|c|c|c|c|}
\hline S. No. & Characteristics & & \\
\hline \multirow[t]{4}{*}{1} & Vegetation & Numberof Species & Percentage \\
\hline & Families & 60 & - \\
\hline & Genera & 164 & - \\
\hline & Species & 238 & - \\
\hline \multirow[t]{5}{*}{2} & Habitat type & Number of Species & Percentageof species \\
\hline & Dry places & 115 & 48.31 \\
\hline & Cultivated & 42 & 17.64 \\
\hline & Wet and dry places & 46 & 19.32 \\
\hline & Wet places & 35 & 14.70 \\
\hline \multirow[t]{4}{*}{3} & Habit & Number of Species & Percentageof species \\
\hline & Herbs & 167 & 70.16 \\
\hline & Trees & 43 & 18.06 \\
\hline & Shrubs & 28 & 11.76 \\
\hline \multirow[t]{5}{*}{4} & Seasonality & Number of Species & Percentage of species \\
\hline & Summer & 185 & 30.57 \\
\hline & Spring & 182 & 30.08 \\
\hline & Autumn & 130 & 21.48 \\
\hline & Winter & 108 & 17.85 \\
\hline \multirow[t]{10}{*}{5} & Life form classes & Number of Species & Percentage of species \\
\hline & Therophytes & 102 & 42.85 \\
\hline & Hemicryptophytes & 25 & 10.50 \\
\hline & Nanophanerophytes & 27 & 11.34 \\
\hline & Chamaephytes & 20 & 8.40 \\
\hline & Microphanerophytes & 18 & 7.56 \\
\hline & Megaphanerophytes & 16 & 6.72 \\
\hline & Geophytes & 15 & 6.30 \\
\hline & Mesophanerophytes & 14 & 5.88 \\
\hline & Parasite & 1 & 0.42 \\
\hline \multirow[t]{8}{*}{6} & Leaf size spectrum & Number of Species & Percentage of species \\
\hline & Microphyll & 73 & 30.67 \\
\hline & Mesophyll & 69 & 28.99 \\
\hline & Nanophyll & 60 & 25.21 \\
\hline & Leptophyll & 23 & 9.66 \\
\hline & Macrophyll & 8 & 1.26 \\
\hline & Megaphyll & 4 & 1.68 \\
\hline & Aphyllous & 1 & 0.42 \\
\hline \multirow[t]{7}{*}{7} & Lamina shape & Number of Species & Percentage of species \\
\hline & Simple & 154 & 64.70 \\
\hline & Compound & 37 & 15.54 \\
\hline & Dissected & 37 & 15.54 \\
\hline & Spiny & 5 & 2.10 \\
\hline & Absent & 3 & 1.26 \\
\hline & Needles & 2 & 0.84 \\
\hline
\end{tabular}


468 Muhammad Idrees, Wisal Muhammad Khan et al. Phytoclimatic Spectrum ....

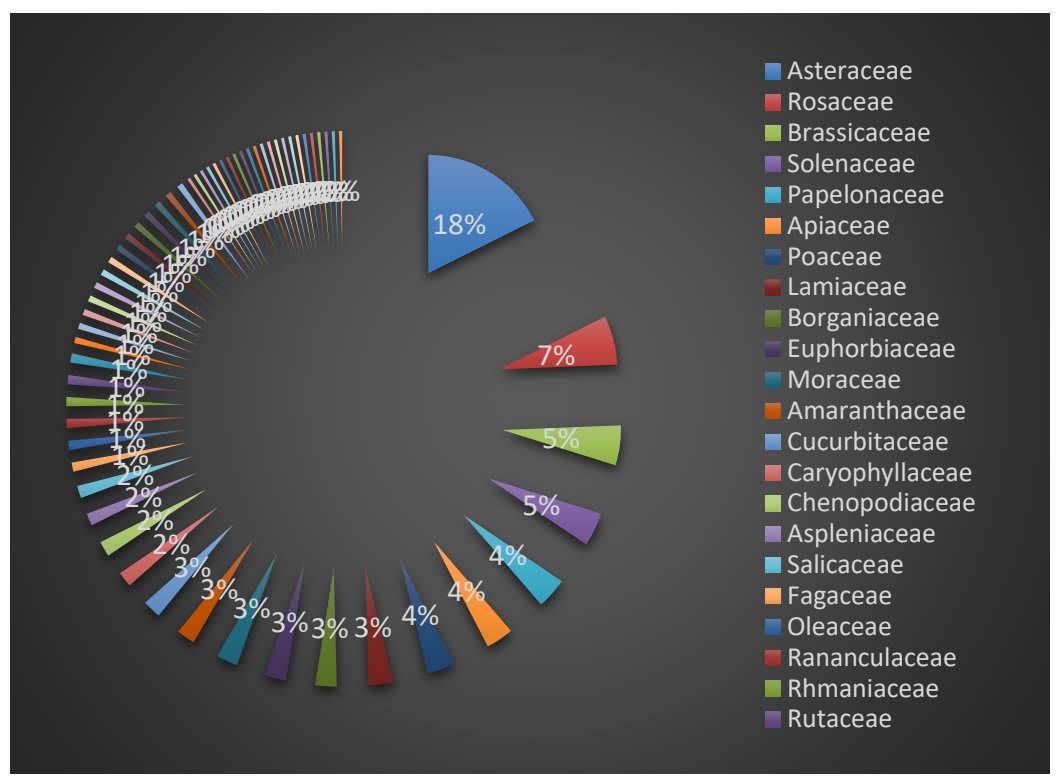

Fig 1. Percentage of plant species

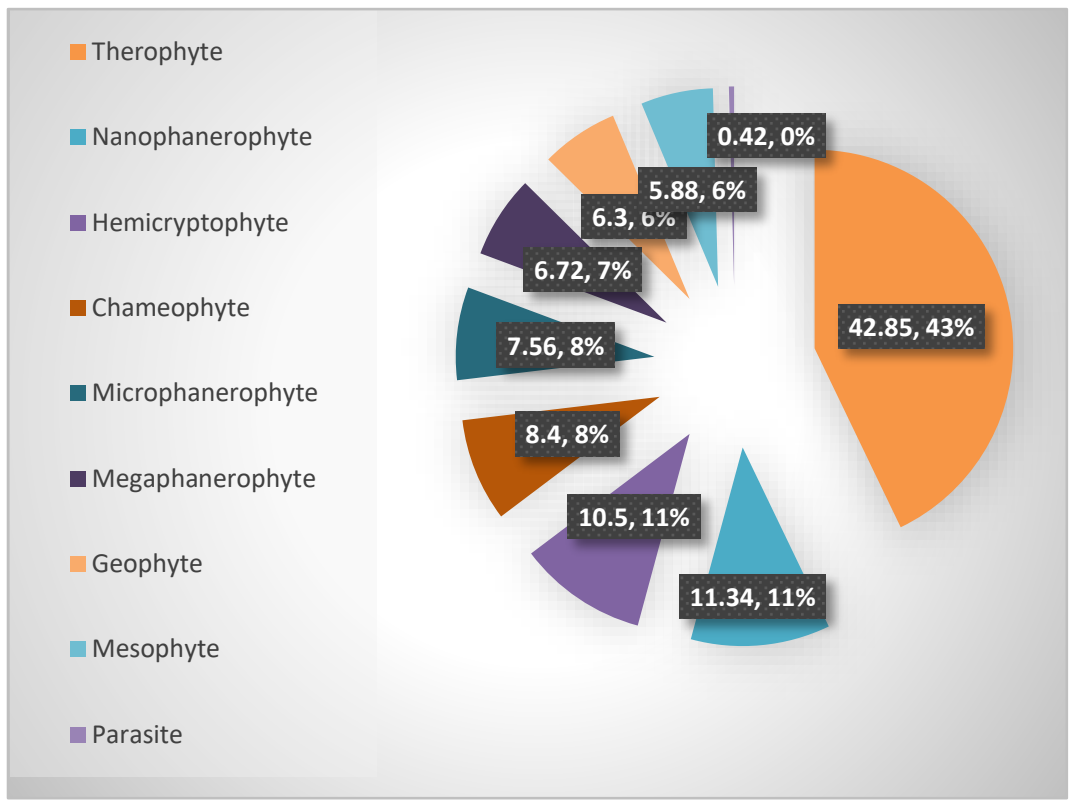

Fig 2. Life form classes 


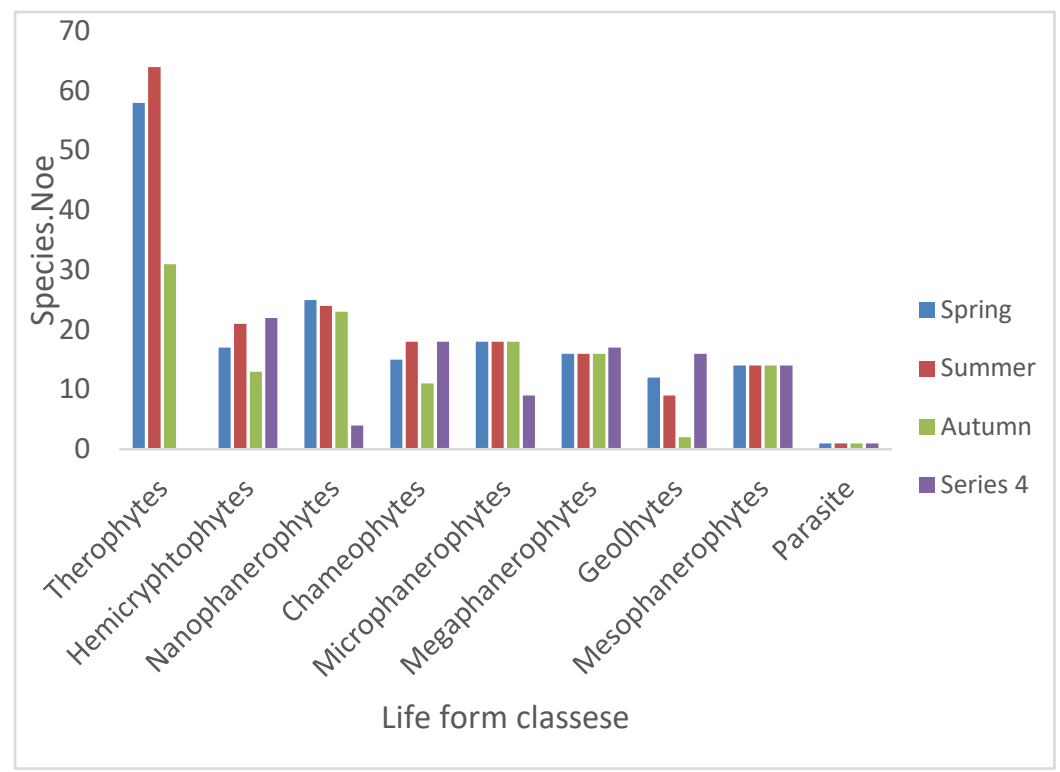

Fig 3. Seasonal variation in the life form of vegetation.

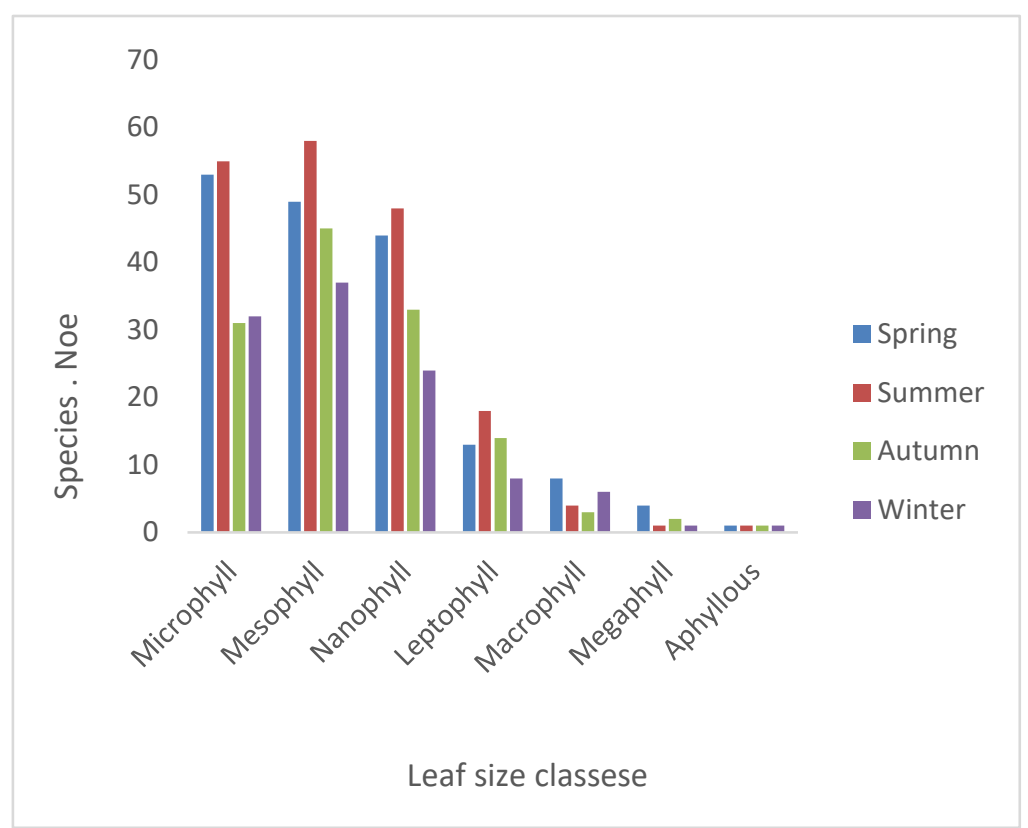

Fig 4. Seasonal variation in leaf size spectrum. 


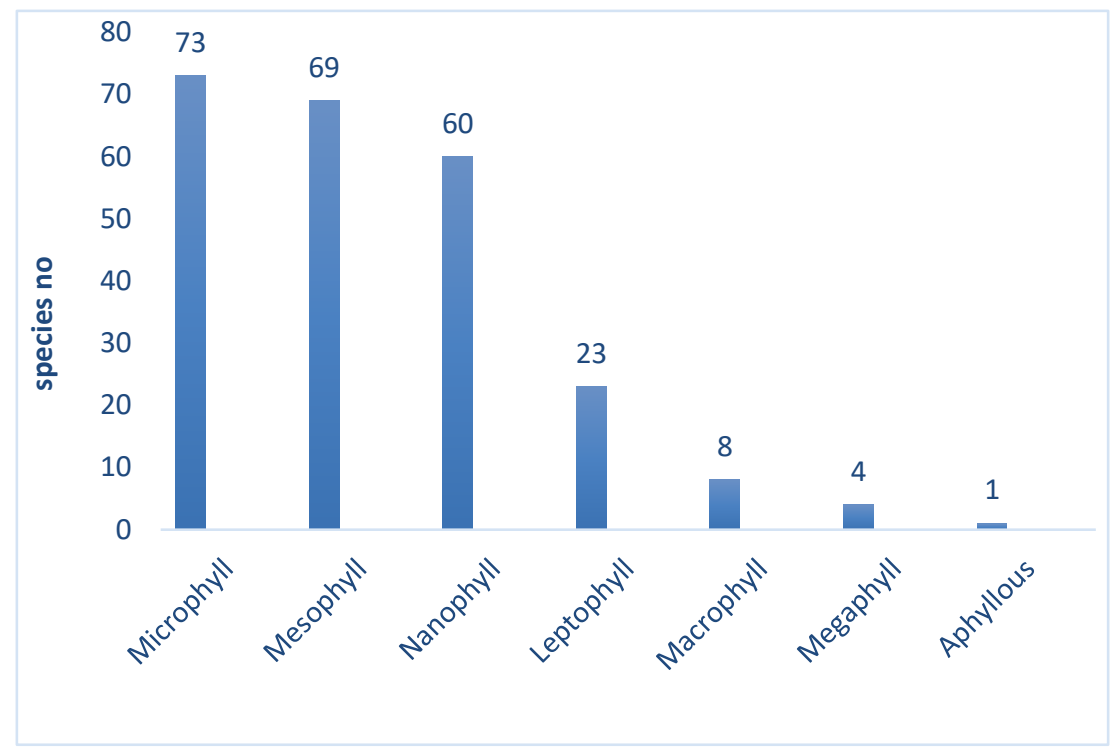

Fig 5. Percentage of species based on vegetation.

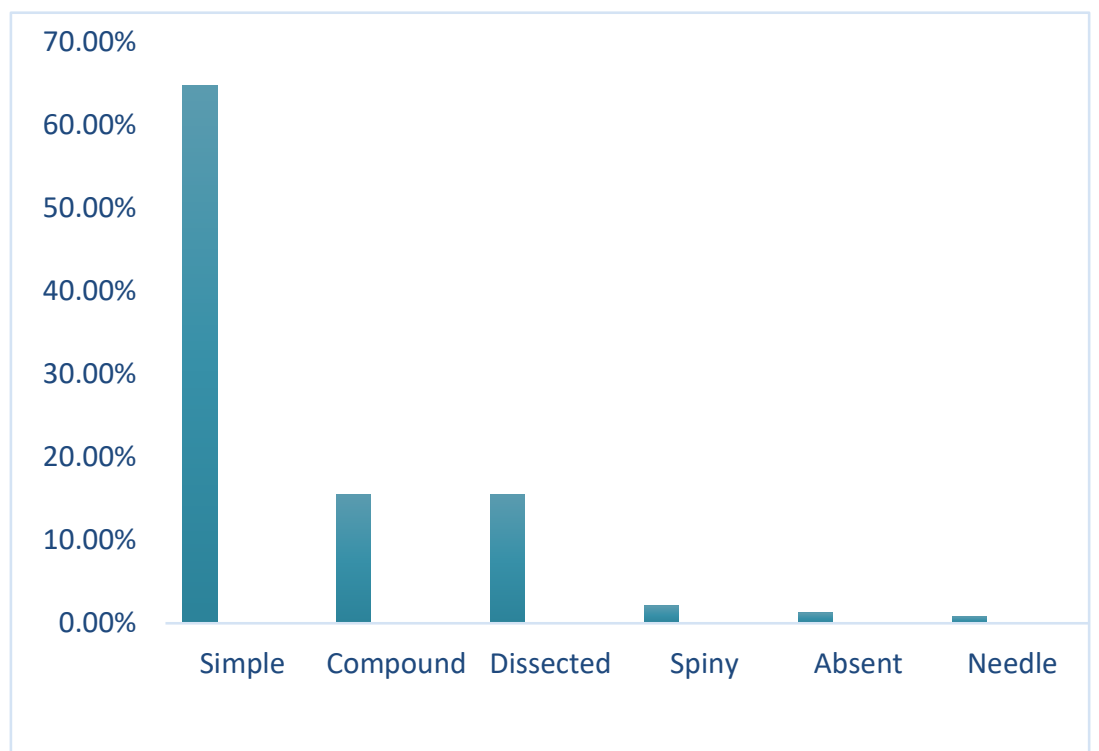

Fig 6. Leaf size spectrum of lamina shape. 


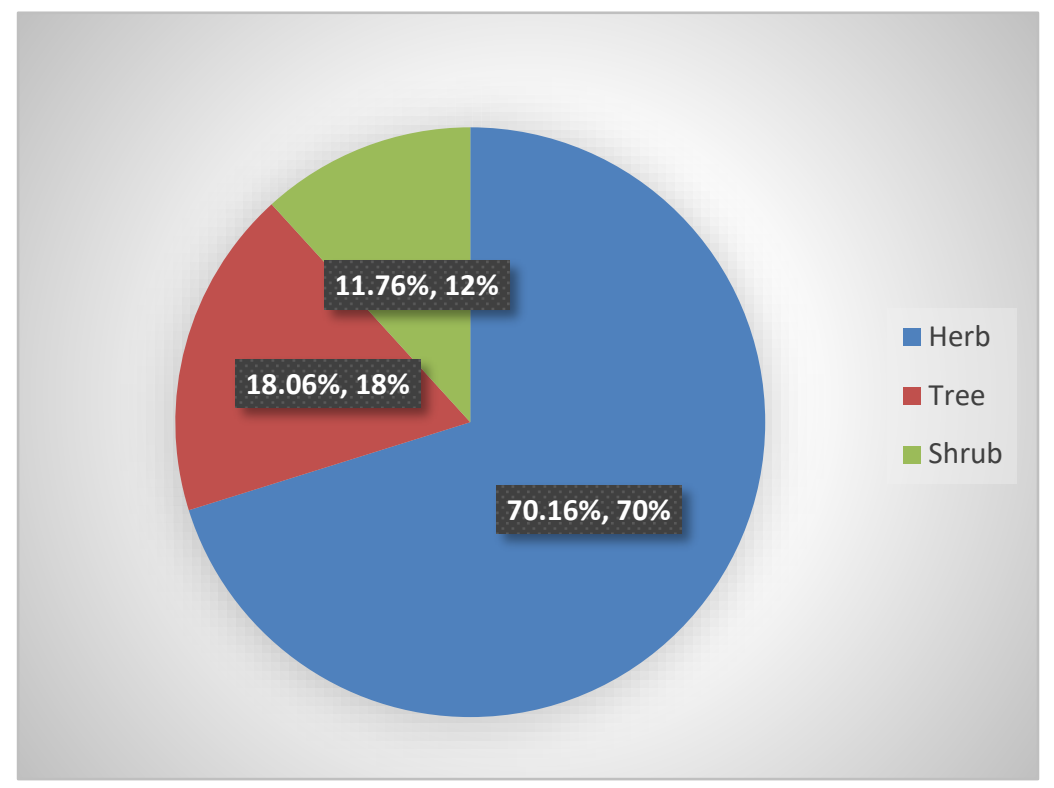

Fig 7. Percentage of species based on their habitat

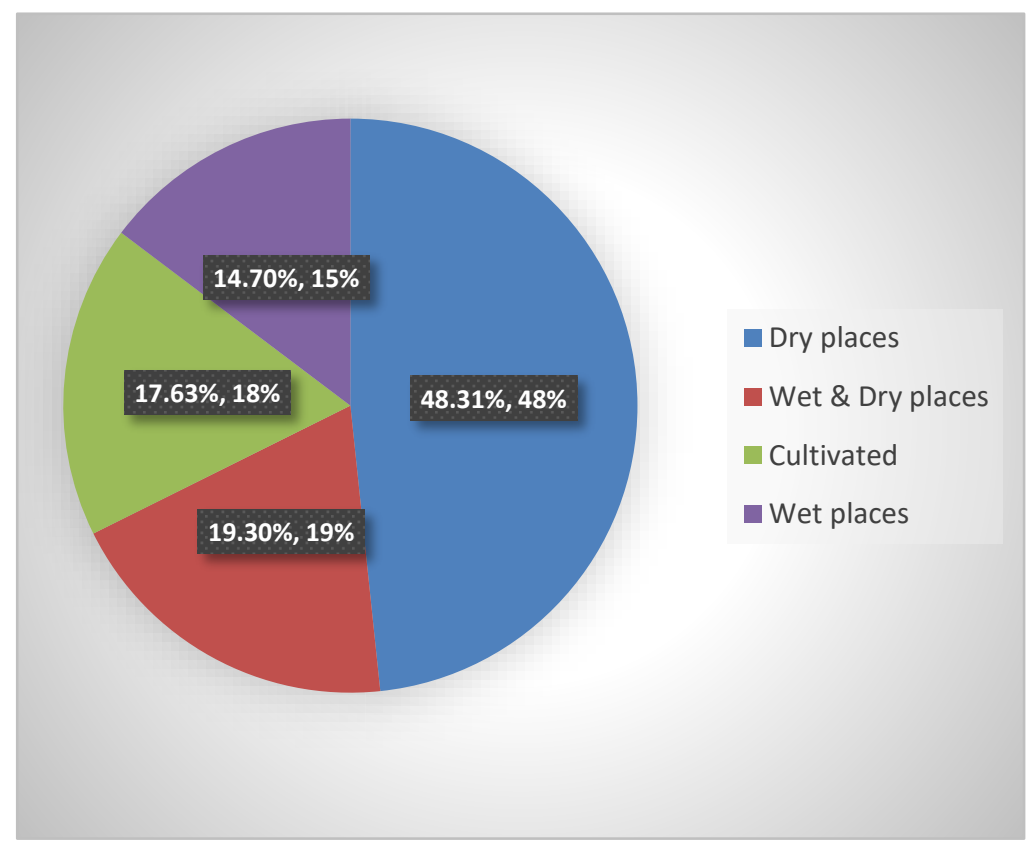

Fig 8. Percentage of species based on their habit 


\section{REFERENCES CITED}

Ahmad, S.S., and H. Ehsan. 2012. Analyzing the herbaceous flora of Lohi Bher Wildlife Park under variable environmental stress. Pak. J. Bot., 44(1): 11-14.

Ali, S., M. Musa, Z. Hussains. Shah, S. Uddin and W. Khan. 2016. Ethnobotanical study of weeds at Mohmand agency, Pakistan. Pak. J. Weed Sci. Res., 22(3): 491498.

Ali, S.I. 2008. The significance of flora with special reference to Pakistan. Pak. J. Bot., 40(30): 967-971.

Alsherif, E.A., A.M. Ayesh and S.M. Rawi. 2013. Floristic composition, life form and chorology of plant life at Khulais region, Western Saudi Arabia. Pak. J. Bot., 45(1): 29-38.

Amjad, M.S. 2012. Life form and leaf size spectra of vegetation in Kotli Hills, Azad Jammu and Kashmir (Pakistan). Greener J. of Agric. Sci., 2(7): 345-350.

Badshah, L., F. Hussain and Z. Sher. 2016. Floristic inventory, ecological characteristics \& biological spectrum of plants of Parachinar, Kurram Agency, Pakistan. Pak. J. Bot., 48(4): 1547-1558.

Ganji, E. 2016. Flora, life form and vegetation structure geographical distribution of plants in Mining in the West of Iran. J. Appl. Environ. Biol. Sci., 6(2): 141146.

Haq, F.U., H. Ahmad, M. Alam, I. Ahmad and Rahatullah. 2010. Species diversity of vascular plants of Nandiar Valley Western Himalaya, Pakistan. Pak. J. Bot., 42(SI): 213-229.

Hussain, M. 2003. The exploitation of legume diversity indigenous to the Salt Range in Punjab. Annual technical report submitted to PARC Islamabad, Pakistan.

Hussain, F., M. Shah, L. Badshah, and M.J. Durrani. 2015. Diversity and ecological characteristics of flora of Mastuj Valley, District Chitral, Hindukush range, Pakistan. Pak. J. Bot., 47(2): 495-510.
Inayat, N., U. Asad and R. Abdur 2014. Floristic composition and ecological prevalence of the weed species growing in wheat and sugar cane fields of District Charsadda, Khyber Pakhtunkhwa. Pak. J. Weed Sci. Res., 20(3): 405-415.

Khan, M., S. Musharaf, and Z. K. Shinwari. 2011. Ethnobotanical importance of halophytes of Noshpho salt mine, District Karak, Pakistan. Res. Pharm. Biotecnol., 3(4): 46-52,

Khan, M., F. Hussain and S. Musharaf. 2012. Biological characteristics of plant species in Tehsil Takht-eNasrati, Pakistan. J. Biodiver. Environ. Sci., 2(3): 42-47.

Khan, M., F. Hussain and S. Musharaf. 2013. Floristic composition and biological characteristics of the Vegetation of Sheikh Maltoon Town District Mardan, Pakistan. Ann. Rev. Res. Biol., 3(1): 31-41.

Khan, M., F. Hussain and S. Musharaf. 2014. Floristic composition and ecological characteristics of Shahbaz Garhi, District Mardan, Pakistan. Glob. J. Sci. Front. Res., 14(1): 7-17.

Nasir, E. and S.I. Ali. 1970-89. Flora of Pakistan. No. 1-190. Islamabad, ISL, Pakistan: The University of Karachi and National Herbarium, PARC.

Naveed, S., F. Hussain, I. Khattak, and L. Badshah. 2012. Floristic composition and ecological characteristics of Olea-Acacia Forest of Shamshokii District Karak. Glob. J. Sci. Front. Res., 12 (8): 31-36.

Nazar, R., S. Begum, A. Naz, R. Qureshi, R. A. Memon, A.K. Chaudhry and Z. Akram 2008. Weed flora of Pir Mehr Ali Shah Agriculture University Rawalpindi: Winter Aspect. Pak. J. Weed Sci. Res., 14(1-2): 55-72.

Oosting, H.J. 1956. The study of plant communities, 2nd edition, W.H. Freeman and company, SanFrancisco, California, USA. p: 440. 
Rafay, M., R.A. Khan, S. Yaqoob and M. Ahmad. $2013 . \quad$ Floristic composition of grass species in the degrading rang lands of Cholistan Desert. Pak. J. Agric. Sci., 50(4): 599-603.

Raunkiaer, C.1934. The life forms of plants and statistical plants geography being the collected papers of C. Raunkiaer. Clarendon on the press, Oxford.

Ravanbakhsh, M. and T. Amini 2014. A Study on floristic composition, chorology, and ecological structure: A case study from a small-scale forest reserve, Talesh, Iran. Eur. J. Biol., 73(1): 43-51.

Samreen, U., M. Ibrar and L. Badshah. 2016. Floristic composition, ecological characters, and Biological characters of Dara Zinda F.R., D. I. Khan, Pakistan. Int. Inv. J. Agric Soil Sci., 4(1): 9-21.

Seraj, S.S., R.N. Jrais and S.K. Ayyad. 2014. Floristic composition, life form and chorology of plant life at Al-Saoda, Asir Region, SouthWestern Saudi Arabia. J. Biol. Agric. Healthcare., 4(26): 60-65.

Shah, M., F. Hussain, S. N. Shah, I.Ahmad and H. Wasila. 2013. Life form and floristic characteristics along altitudinal Gradient of Humid Temperate Forests located in Remote Area of Pakistan. Glob. J. Biol. Divers. Sci. Manage., 3(2): 276-281,

Sher, Z. and Z.U. Khan. 2007. Floristic composition, life form and leaf spectra of the vegetation of Chagharzai Valley, District Buner. Pak. J. Plant Sci., 13(1): 55-64.

Sher, Z., F. Hussain and L. Badshah. 2014. Biodiversity and ecological characterization of the flora of Gadoon Rangeland, District Swabi, Khyber Pakhtunkhwa, Pakistan. Iran. J. Bot., 20(1): 96108.

Shimwell, D. W. 1971. The Description and Classification of Vegetation Sedgwick and Jackson, London. pp. 322.

Ullah, A. and S. Ullah. 2016. Vascular plant diversity in Landi Kotal
Valley, Khyber Agency, Pakistan. Fuuast J. Biol., 6(2): 265-271.

Ullah, S. and L. Badshah. 2017. Floristic structure and ecological attributes of Jelar Valley flora, District Upper Dir, Pakistan. J. Bio. Env. Sci., 10 (5): 89-105. 